\title{
Klein tunneling of optically tunable Dirac particles with elliptical dispersions
}

\author{
Andrii Iurov $\odot,{ }^{1, *}$ Liubov Zhemchuzhna $\odot,{ }^{2,1}$ Paula Fekete $\odot,{ }^{3}$ Godfrey Gumbs $\odot,{ }^{2,4}$ and Danhong Huang $\oplus^{5,6}$ \\ ${ }^{1}$ Department of Physics and Computer Science, Medgar Evers College of the City University of New York, Brooklyn, New York 11225, USA \\ ${ }^{2}$ Department of Physics and Astronomy, Hunter College of the City University of New York, 695 Park Avenue, New York, New York 10065, USA \\ ${ }^{3}$ US Military Academy at West Point, 606 Thayer Road, West Point, New York 10996, USA \\ ${ }^{4}$ Donostia International Physics Center (DIPC), P de Manuel Lardizabal, 4, 20018 San Sebastian, Basque Country, Spain \\ ${ }^{5}$ US Air Force Research Laboratory, Space Vehicles Directorate, Kirtland Air Force Base, New Mexico 87117, USA \\ ${ }^{6}$ Center for High Technology Materials, University of New Mexico, 1313 Goddard SE, Albuquerque, New Mexico, 87106, USA
}

(Received 27 June 2020; accepted 19 October 2020; published 17 November 2020)

\begin{abstract}
We have investigated electron tunneling through an atomically smooth square potential barrier for both the dice lattice and graphene under a linearly polarized off-resonant and high-frequency dressing field. We have demonstrated Klein tunneling for a nonzero angle of incidence which is due to a nonalignment of optically controllable elliptical energy dispersions for the dressed states of Dirac particles and the direction of incoming kinetic particles. This finite angle of incidence has been found to depend on the light-induced anisotropy of energy dispersion, which is a function of the electron-light coupling strength, as well as the misalignment between directions of the light polarization and the electron beam incident on the potential barrier. Additionally, we have discovered much larger off-peak transmission amplitudes for dice lattices in contrast to graphene. We anticipate that the theoretical predictions could be applied to a wide range of Dirac materials and exploited for controlling both coherent tunneling and ballistic transport of electrons in the construction of novel electronic, optical, and valleytronic nanoscale switching devices.
\end{abstract}

DOI: 10.1103/PhysRevResearch.2.043245

\section{INTRODUCTION}

The $\alpha-\mathcal{T}_{3}$ model is one of the most recent and a very promising system with zero-mass Dirac fermions [1]. Unlike graphene [2,3], the low-energy electronic states of $\alpha-\mathcal{T}_{3}$ lattices are governed by a $3 \times 3$ pseudospin- 1 Hamiltonian and described mathematically by pseudospin-1 Dirac-Weyl equation [4-7]. The resulting energy dispersion is distinguished by the presence of a completely flat band with infinite degeneracy exactly at the Dirac point, and also acquires a Dirac cone structure as in graphene simultaneously. These Dirac cone bands could open an energy gap or display an anisotropy if external irradiation with a proper polarization is applied to the system [8,9].

The lattice structure for the $\alpha-\mathcal{T}_{3}$ model simply consists of a honeycomb lattice of atoms plus an additional hub atom at the center of each hexagon. This hub atom couples to one of the $A$ - or $B$-sublattice atoms on the rim with its hopping coefficient equal to a fraction of that between two neighboring sublattice atoms on the hexagon rim sites. This ratio $\alpha$ varies from 0 , which is equivalent to the model for graphene with a completely decoupled set of hub atoms, to 1, corresponding to a dice lattice in which the influence of the extra hub atom reaches a maximum.

\footnotetext{
*aiurov@mec.cuny.edu; theorist.physics@gmail.com
}

Published by the American Physical Society under the terms of the Creative Commons Attribution 4.0 International license. Further distribution of this work must maintain attribution to the author(s) and the published article's title, journal citation, and DOI.
There has been a great deal of encouraging experimental evidence for the fabrication [10-14] of dice or $\alpha-\mathcal{T}_{3}$ lattices based on various atomic and electronic properties of some known materials [15-19]. One of the most well-known and widely discussed candidates is the three-layer arrangement of $\mathrm{SrTiO}_{3} / \mathrm{SrIrO}_{3} / \mathrm{SrTiO}_{3}$ lattices in which each of three initial layers possesses a cubic crystal structure. A particularly useful and complete review on the experimentally fabricated flat band materials can be found in Ref. [20] and the cited references therein.

So far, novel $\alpha-\mathcal{T}_{3}$ model has exhibited nontrivial topological properties [21-24] related to its band structure. This is due to the presence of a flat band. Also, unique topological features have been displayed in many of its physical properties [9,25-30], including both optical [4,31] and magnetic [32-35] ones. One fascinating behavior exhibited is the phase transition from a diamagnetic to paramagnetic phase transition under a perpendicular quantizing magnetic field as the $\alpha$ parameter increases from zero [36,37]. Meanwhile, the electronic [21,38], collective [5,31], and transport [39-41] phenomena in $\alpha-\mathcal{T}_{3}$ lattices are found unique and remarkable. Especially, $\alpha-\mathcal{T}_{3}$ materials allow for regular Klein paradox, [42,43], i.e., unimpeded tunneling for Dirac electrons incident perpendicular to a sharp, atomically smooth potential barrier $[44,45]$, just as was observed earlier for graphene [46-50]. Interestingly, such perfect transmission is independent of the barrier height and width. Klein birefringent tunneling was also demonstrated for spin-3/2 Dirac fermions with a double-layered Dirac cone $s$ in Refs. [51,52].

The electronic states and their properties in all these twodimensional (2D) materials could be physically modified and 
even fine tuned effectively by employing the so-called Floquet engineering, i.e., applying an off-resonant and high-frequency dressing field with various polarizations. The practical use of such a semiclassical dressing approach with a nonionizing but intense laser field has only become possible over the past several years due to significant progress made in microwave, laser, and teraherz technologies. The modification of electronic properties based on external irradiation has been addressed theoretically in an extensive way with the help from Floquet theory [53-56] for periodically driven quantum systems [57], covering a wide range of 2D materials [58,59] such as graphene $[8,60]$, silicene and transitional-metal dichalcogenides [61,62], phosphorene [63], and purely quantum-spin systems [64] as well as on the surfaces of three-dimensional topological insulators $[65,66]$.

The effect due to external radiation on a $2 \mathrm{D}$ material depends crucially on its polarization. Circularly polarized light opens a band gap between the valence and conduction bands of an isotropic energy spectrum [8] as well as suppression of Klein tunneling [67,68]. The opened band gap plays an important role on collective charged carrier properties in $\alpha-\mathcal{T}_{3}$ lattices $[69,70]$. In contrast, linearly polarized irradiation creates an anisotropy in the Dirac dispersion [60] or modifies the existing anisotropy within the phosphorene band structure $[63,71,72]$, which is equivalent to applying the most general elliptically polarized dressing field with combined anisotropies from both material band structure and external light-field polarization.

The rest of the paper is organized as follows. In Sec. II, we provide an alternative derivation for the dressed electronic states in a dice lattice using a rather straightforward Floquet-Magnus perturbative expansion for an off-resonant and high frequency periodic dressing field. These electronic states obtained in Sec. II are further compared with results derived in our previous work [9] based on a rigorous analytical solution for $\boldsymbol{k}=0$, followed by an expansion with respect to this complete set for other finite $\boldsymbol{k}$ vectors. The corresponding derivation of such dressed states for arbitrary direction of linear polarization is briefly discussed in Appendix B. Equipped with acquired dressed states of electrons, we investigate electron tunneling dynamics through a square-barrier potential under an external linearly polarized dressing field for both graphene and dice lattice. We demonstrate in Appendix D that the boundary conditions for a dice lattice will be modified substantially as long as the direction of light polarization and that of head-on incidence is not aligned. This is related to the so-called anomalous Klein paradox. We compute electron tunneling in a dice lattice or graphene with an anisotropic Dirac cone in Sec. III and reveal key information on Klein tunneling. In Sec. IV, we analyze and discuss properties of numerical results on electron transmission and reflection in both irradiated graphene and dice lattice, and finally draw conclusions along with remarks in Sec. V.

\section{ELECTRON-DRESSED STATES UNDER LINEARLY POLARIZED IRRADIATION}

In this section, we present an alternative but simplified derivation of electron dressed states in the presence of external linearly polarized irradiation. Using the Floquet-Magnus perturbation expansion aimed to off-resonant dressing field with frequency satisfying $\hbar \omega \gg \mathcal{E}_{0}$, the characteristic energy of electrons, we obtain the quasiparticle energy dispersion relation and closed-form analytic expression for dressed electron wave functions. Even though this paper focuses on two opposite limits for graphene with $\alpha=0$ as well as dice lattice with $\alpha=1$, we still present relevant discussions on properties pertaining to the general $\alpha-\mathcal{T}_{3}$ model.

The starting point is the low-energy Hamiltonian for $\alpha-$ $\mathcal{T}_{3}$ materials under applied linearly polarized radiation with a vector potential $\boldsymbol{A}^{(L)}(t)$ and an electrostatic barrier potential [43] $V(x)=V_{B} \Theta(x) \Theta\left(W_{B}-x\right)$, namely,

$$
\hat{\mathbb{H}}_{0}^{\tau}(\phi \mid x, y)=v_{F} \hat{\boldsymbol{S}}(\phi) \cdot\left\{-i \hbar \nabla_{\{\tau\}}-e \boldsymbol{A}^{(L)}(t)\right\}+V(x),
$$

where $V_{B}$ and $W_{B}$ are the height and width of an atomically smooth potential barrier, $\nabla_{\{\tau\}}=\{\tau \partial / \partial x, \partial / \partial y\}$ is a partial derivative operator which depends on the valley index $\tau= \pm 1$.

The $\phi$-dependent matrices $\hat{\boldsymbol{S}}(\phi)=\hat{S}_{x, y}(\phi)$ employed for constructing the principal Hamiltonian in Eq. (1) are given by

$$
\hat{S}_{x}(\phi)=\left[\begin{array}{ccc}
0 & \cos \phi & 0 \\
\cos \phi & 0 & \sin \phi \\
0 & \sin \phi & 0
\end{array}\right]
$$

and

$$
\hat{S}_{y}(\phi)=i\left[\begin{array}{ccc}
0 & -\cos \phi & 0 \\
\cos \phi & 0 & -\sin \phi \\
0 & \sin \phi & 0
\end{array}\right] .
$$

It is straightforward to verify that the two matrices presented above reduce, respectively, to the well-known spin-1/2 Pauli forms for graphene in the limit of $\phi \rightarrow 0$, and to the symmetric spin-1 ones $\hat{\Sigma}_{x}^{(1)}$ and $\hat{\Sigma}_{y}^{(1)}$ presented in Eqs. (A1) and (A2) in Appendix A for the dice lattice in the limit of $\phi \rightarrow \pi / 4$.

The time-independent potential $V(x)$ introduced in Eq. (1) only depends on the $x$ coordinate but not on the variable $y$. We assume that $V(x)$ is a piecewise-constant profile as commonly employed for studying Klein tunneling $[42,43,46]$. Also, we impose two boundary conditions associated with $V(x)$ at its two edges, preserving translational symmetry along the $y$ direction. On the other hand, the physical characteristics of the carriers, i.e., electrons or holes, within the barrier region are determined by the sign of $\mathcal{E}_{0}-V_{0}$, where $\mathcal{E}_{0}$ represents the kinetic energy of incoming electrons. Since we consider a positive barrier with $V_{B}>0$, there exists only one transition in the barrier region, i.e., electron $\rightarrow$ hole, under the assumption that $V_{B}>\mathcal{E}_{0}$, as illustrated in Fig. 1 .

A possible experimental realization for such an arrangement is important and should be pursued at this point. Most previously reported experimental setups for measuring charge transport through potential barriers in graphene [73] employed an array of tapered electrodes mounted on the upper side of the graphene sheet, while the interface side of the arrangement consists of several silicon-based substrates.

Technological advances involved in these experiments are being rapidly made at the present time. In a recent paper [74], a Klein tunneling experiment was proposed for characterizing the ballistic transport within an Aharonov-Bohm graphene ring with multigates acting as tunable $p n$ junctions in one of its arms. The main idea in this paper is that one of the 


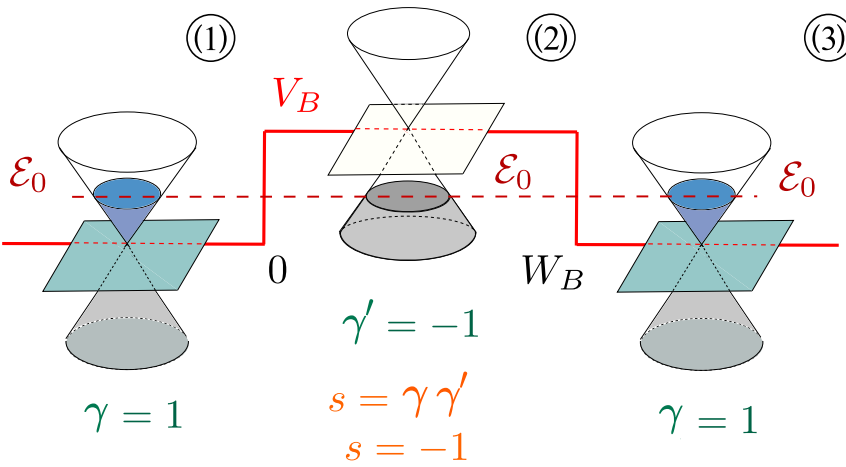

FIG. 1. Schematic illustrations of an incident electron with kinetic energy $\mathcal{E}_{0}$ tunneling through a rectangular potential barrier $V(x)=V_{B} \Theta(x) \Theta\left(W_{B}-x\right)$, where $\Theta(x)$ is the Heaviside unit step function. We have chosen the barrier height $V_{B}$ such that $0<\mathcal{E}_{0}<V_{B}$ and the electron-hole-electron transition only occurs between two edges, $x=0$ and $x=W_{B}$, of the barrier region-2, which is equivalent to an $n$ - $p$ - $n$ multijunction. In this notation, $\gamma=+1$ (or -1 ) refers to the Fermi energy located within the upper (lower) Dirac cone. Additionally, the unit of energy for $\mathcal{E}_{0}$ and $V_{B}$ is the Fermi energy $E_{F}^{(0)}$, while the unit of length for $W_{B}$ is $1 / k_{F}^{(0)}$, where $k_{F}^{(0)}=\sqrt{\pi n_{0}}$ is the Fermi wave number and $E_{F}^{(0)}=\hbar v_{F} k_{F}^{(0)}$ with $v_{F}$ and $n_{0}$ denoting the Fermi velocity and areal doping density, respectively.

gates was located above the source of the external magnetic field away from the active 2D layer. Similarly, the top gate in our design could also be placed above the local source for electromagnetic radiation.

By taking $V(x)=0$ in Eq. (1), due to the presence of translational symmetry in this system, we acquire a simple relation, i.e., $\{\partial / \partial x, \partial / \partial y\} \rightarrow i\left\{k_{x}, k_{y}\right\}$, for the wave function $\Psi(x, y) \backsim e^{i k_{x} x} e^{i k_{y} y}$, and then the first term of the Hamiltonian in Eq. (1) becomes

$\hat{\mathcal{H}}_{\alpha}(\boldsymbol{k} \mid \tau, \phi)=\hbar v_{F}\left[\begin{array}{ccc}0 & k_{-}^{\tau} \cos \phi & 0 \\ 0 & 0 & k_{-}^{\tau} \sin \phi \\ 0 & 0 & 0\end{array}\right]+$ H.c.,

where $k_{ \pm}^{\tau}=\tau k_{x} \pm i k_{y}$ depends on the valley index $\tau= \pm 1$. Here, the geometry phase $\phi$ is related to the ratio of the hopping amplitudes $\alpha$ by $\phi=\tan ^{-1} \alpha$ for $0 \leqslant \phi \leqslant \pi / 4$ (later we will only consider a dice lattice with $\alpha=1$ or $\phi=\pi / 4$ ) and + H.c. means adding a Hermitian conjugate of the first term. From now on, we will only consider a dice lattice with $\alpha=1$ or $\phi=\pi / 4$.

Particularly, for the case of a dice lattice, the Hamiltonian in Eq. (4) for $V(x)=0$ is simplified as

$$
\hat{\mathcal{H}}_{1}(\boldsymbol{k} \mid \tau)=\frac{\hbar v_{F}}{\sqrt{2}}\left[\begin{array}{ccc}
0 & k_{-}^{\tau} & 0 \\
k_{+}^{\tau} & 0 & k_{-}^{\tau} \\
0 & k_{+}^{\tau} & 0
\end{array}\right]=\frac{\hbar v_{F}}{2} \sum_{\mu= \pm} \hat{\Sigma}_{-\mu}^{(1)} k_{\mu}^{\tau},
$$

where $\hat{\Sigma}_{ \pm}^{(1)}$ are derived and explained in Eqs. (A5) and (A6) of Appendix A.

The main goal of this paper is to determine the electron dressed states under a linearly polarized dressing field. We assume the polarization of the dressing field lies in the $x$ direction, while the general case with an arbitrary polarization direction is addressed in Appendix B. Under this assumption, the vector potential takes the form

$$
\boldsymbol{A}^{(L)}(t)=\left[\begin{array}{l}
A_{x}^{(L)}(t) \\
A_{y}^{(L)}(t)
\end{array}\right]=\frac{E_{0}}{\omega}\left[\begin{array}{l}
1 \\
0
\end{array}\right] \cos (\omega t) .
$$

This is one limiting case of the most general elliptically polarized light, while the opposite limit of two equal components of the vector potential corresponds to a circular type of light polarization. For any type of external dressing field, its wave vector $\boldsymbol{k}$ in the Hamiltonian in Eq. (5) will be modified according to the canonical substitution, $k_{x, y} \rightarrow k_{x, y}-e A_{x, y} / \hbar$.

Since the Hamiltonian in Eq. (5) is linear in $k_{x, y}$, in the presence of $\boldsymbol{A}^{(L)}(t)$, it only acquires an additional interaction term, which yields

$$
\hat{\mathcal{H}}_{1}(\boldsymbol{k} \mid \tau) \Longrightarrow \hat{\mathbb{H}}^{(L)}(\boldsymbol{k}, t)=\hat{\mathcal{H}}_{1}(\boldsymbol{k} \mid \tau)+\hat{\mathcal{H}}_{A}^{(L)}(t),
$$

where the $\boldsymbol{k}$ independent interaction term is

$$
\hat{\mathcal{H}}_{A}^{(L)}(t)=-\frac{\tau c_{0}}{\sqrt{2}} \cos (\omega t)\left[\begin{array}{lll}
0 & 1 & 0 \\
1 & 0 & 1 \\
0 & 1 & 0
\end{array}\right] .
$$

Here, the optical-coupling strength parameter $c_{0}=e v_{F} E_{0} / \omega$ remains the same for all types of light polarizations, which implies that its effect on the energy dispersions has a similar magnitude but different features. In fact, the time-dependent second term in Eq. (7) is the same for all matrix elements of $\hat{\mathcal{H}}_{1}(\boldsymbol{k} \mid \tau)$, which is unique for the linear type of imposed light polarization.

In this paper, we apply the Floquet-Magnus perturbation approach to the Hamiltonian in Eq. (7) for a high-frequency off-resonant dressing field. To serve this purpose, we first rewrite the time-dependent second term in Eq. (8) into

$$
\hat{\mathcal{H}}_{A}^{(L)}(t)=\hat{\mathbb{O}}_{1}\left(c_{0}, \tau\right) e^{i \omega t}+\hat{\mathbb{O}}_{1}^{\dagger}\left(c_{0}, \tau\right) e^{-i \omega t},
$$

where the time-independent operator $\hat{\mathbb{O}}_{1}\left(c_{0}, \tau\right)$ is defined as

$$
\hat{\mathbb{O}}_{1}\left(c_{0}, \tau\right)=-\frac{\tau c_{0}}{2 \sqrt{2}}\left[\begin{array}{lll}
0 & 1 & 0 \\
1 & 0 & 1 \\
0 & 1 & 0
\end{array}\right]=-\frac{\tau c_{0}}{4}\left(\hat{\Sigma}_{+}^{(1)}+\hat{\Sigma}_{-}^{(1)}\right) .
$$

Next, by employing the high-frequency Floquet-Magnus expansion technique, the time-independent effective part of the total Hamiltonian in Eq. (7) becomes

$$
\begin{aligned}
\hat{\mathcal{H}}_{\mathrm{eff}}^{(L)}(\boldsymbol{k} \mid \tau)= & \hat{\mathcal{H}}_{1}(\boldsymbol{k} \mid \tau)+\frac{1}{\hbar \omega}\left[\hat{\mathbb{O}}_{1}\left(c_{0}, \tau\right), \hat{\mathbb{O}}_{1}^{\dagger}\left(c_{0}, \tau\right)\right] \\
& +\frac{1}{2(\hbar \omega)^{2}}\left\{\left[\left[\hat{\mathbb{O}}_{1}\left(c_{0}, \tau\right), \hat{\mathcal{H}}_{1}(\boldsymbol{k} \mid \tau)\right],\right.\right. \\
& \left.\left.\times \hat{\mathbb{O}}_{1}^{\dagger}\left(c_{0}, \tau\right)\right]+ \text { H.c. }\right\}+\cdots,
\end{aligned}
$$

where the first term in Eq. (11) is just the nonperturbed Hamiltonian in Eq. (5) in the absence of irradiation, and the following term $\left[\hat{\mathbb{O}}_{1}\left(c_{0}, \tau\right), \hat{\mathbb{O}}_{1}^{\dagger}\left(c_{0}, \tau\right)\right]$ is zero since $\hat{\mathbb{O}}_{1}\left(c_{0}, \tau\right)$ is Hermitian. This conclusion holds true only for linearly polarized light in contrast to all other polarizations and cases with a finite band gap. The remaining third term in Eq. (11) is 
written as $\hat{\mathbb{T}}_{2}\left(\lambda_{0} \mid k, \theta_{\mathbf{k}}\right)$ and calculated to give the result

$$
\begin{aligned}
\hat{\mathbb{T}}_{2}\left(\lambda_{0} \mid k, \theta_{\mathbf{k}}\right) & =i \frac{\lambda_{0}^{2}}{4 \sqrt{2}} \hbar v_{F} k_{y}\left[\begin{array}{ccc}
0 & 1 & 0 \\
-1 & 0 & 1 \\
0 & -1 & 0
\end{array}\right] \\
& =-\frac{\lambda_{0}^{2}}{4} \hbar v_{F} k_{y} \hat{\Sigma}_{y}^{(1)},
\end{aligned}
$$

where $\lambda_{0}=c_{0} / \hbar \omega$ is a dimensionless light-matter interaction parameter.

The Floquet-Magnus effective Hamiltonian in Eq. (11) is time independent, leading to time-independent eigenvalues, wave functions, as well as new boundary conditions derived from $\hat{\mathbb{T}}_{2}\left(\lambda_{0} \mid k, \theta_{\mathbf{k}}\right)$. The obtained eigenvalues could be regarded as time averaged with respect to a fast oscillating behavior in the off-resonant limit (i.e., neglecting Rabi oscillations with a very short period and a very small amplitude), and the fast-oscillating interaction Hamiltonian in Eq. (9) only acquires a factor of $e^{ \pm i \omega t}$ for its time dependence subject to the weak-field approximation with $\hbar \omega \gg c_{0}$ (i.e., neglecting photon-assisted electron tunneling.

Once the total Hamiltonian for dressed state electrons is obtained, we are able to solve the corresponding eigenvalue equation to obtain the energy dispersion relation. In addition to the flat band $\varepsilon^{\gamma=0}\left(\lambda_{0}, \boldsymbol{k}\right)=0$, we also have two other energy bands

$$
\varepsilon_{1}^{\gamma= \pm 1}\left(\lambda_{0}, \boldsymbol{k}\right)=\gamma \hbar v_{F} \sqrt{k_{x}^{2}+\left(1-\frac{\lambda_{0}^{2}}{4}\right)^{2} k_{y}^{2}}
$$

for the valence $(\gamma=-1)$ and conduction $(\gamma=+1)$ dressed states. We now introduce an anisotropic-dispersion factor $a_{1}\left(\lambda_{0}\right)$ defined by $\varepsilon_{1}^{\gamma= \pm 1}\left(\lambda_{0}, \boldsymbol{k}\right)= \pm \hbar v_{F} \sqrt{k_{x}^{2}+a_{1}^{2}\left(\lambda_{0}\right) k_{y}^{2}}$ and find

$$
a_{1}\left(\lambda_{0}\right)=1-\frac{\lambda_{0}^{2}}{4} .
$$

Equations (13) and (14) agree with the previous result [9] in the limit of $\phi \Longrightarrow \pi / 4$ (or $\alpha \Longrightarrow 1$ ) for the energy dispersion of an irradiated $\alpha-\mathcal{T}_{3}$ lattice, given by

$$
\begin{aligned}
& \varepsilon_{\alpha}^{\gamma= \pm 1}\left(\lambda_{0}, \boldsymbol{k}\right)=0 \text { and } \\
& \varepsilon_{\alpha}^{\gamma= \pm 1}\left(\lambda_{0}, \boldsymbol{k}\right)= \pm \hbar v_{F} k \sqrt{\mathcal{F}\left(\theta_{\mathbf{k}} \mid \phi, \lambda_{0}\right)},
\end{aligned}
$$

where

$$
\begin{aligned}
\mathcal{F}\left(\theta_{\mathbf{k}} \mid \phi, \lambda_{0}\right)= & \cos ^{2} \theta_{\mathbf{k}}+\left[J_{0}^{2}\left(2 \lambda_{0}\right) \cos ^{2}(2 \phi)\right. \\
& \left.+J_{0}^{2}\left(\lambda_{0}\right) \sin ^{2}(2 \phi)\right] \sin ^{2} \theta_{\mathbf{k}} .
\end{aligned}
$$

Here, $J_{0}(x)$ is the zeroth-order Bessel function of the first kind, and the anisotropic factor of the dispersion is calculated from Eq. (16) as

$$
a_{\alpha}\left(\lambda_{0}\right)=1-\frac{\lambda_{0}^{2}}{8}[5+3 \cos (4 \phi)] .
$$

Clearly, Eqs. (15) and (17) for $\phi=\pi / 4$ exactly match Eqs. (13) and (14) for a dice lattice.

As we display in Figs. 2(d)-2(f), the energy dispersion in Eq. (13) for a dice lattice exhibits an anisotropy due to the applied linearly polarized light. In general, the anisotropic dispersion relation in Eqs. (15) will rely on the phase $\phi$ or parameter $\alpha$ for a general $\alpha-\mathcal{T}_{3}$ lattice, as shown in Figs. 2(a)-2(c). This anisotropic behavior becomes the strongest for graphene but the weakest for a dice lattice by comparing Fig. 2(a) with Fig. 2(e) for fixed $\lambda_{0}=0.3$. Numerically, we can verify that Eq. (13), which is obtained based on the expansion in Eq. (11), demonstrates good accuracy provided $\lambda_{0} \leqslant 0.4$. Furthermore, the anisotropy in energy dispersion becomes visible for $\lambda_{0} \backsim 0.1$ and larger. All the ovals are elongated along the $y$ axis for the light polarization along the $x$ axis, and the anisotropy for the dice lattice is manifested as the eccentricity of dispersion ellipses increases with $\lambda_{0}$ in Figs. 2(d)-2(f).

The calculations of the electron-dressed state dispersion relation and their wave functions were based on the Floquet-Magnus perturbation expansion for the light-electron interaction Hamiltonian. This procedure is applicable in the high-frequency and off-resonance regime for external dressing fields. From a physical point of view, this assumes that the frequency for the impinging linearly polarized field is much larger than any characteristic energy of electrons in considered materials, i.e., the Fermi energy $E_{F}^{(0)}$ and light-electron interaction strength $c_{0}=\left(e v_{F} E_{0}\right) / \omega$. Consequently, there are two relevant quantities, i.e., the intensity of the applied radiation $\mathcal{I}_{0}$ (power of the light beam per unit area) and its frequency $\omega=2 \pi f$, which are required to satisfy this assumption up to a factor of $\times 10$ in an actual experiment.

In a practical experimental setup, we assume $\mathcal{I}_{0}=$ $1 \mathrm{~kW} / \mathrm{cm}^{2}=10^{7} \mathrm{~W} / \mathrm{m}^{2}$ for the irradiation intensity, while the frequency lies in the terahertz range, i.e., $f \backsim 10^{12}-$ $10^{13} \mathrm{~Hz}$ or, equivalently, $\omega \backsim 10^{13}-10^{14} \mathrm{rad} / \mathrm{s}$. For chosen $\mathcal{I}_{0}=10^{7} \mathrm{~W} / \mathrm{m}^{2}$ and a sample area on the order of $\backsim 10^{-15} \mathrm{~m}^{2}$, we obtain the corresponding energy $\mathcal{E}_{\text {ph }}$ for a light beam as $\mathcal{E}_{\mathrm{ph}} \backsim 10^{-21} \mathrm{~J}$, or a few meV. The electric field amplitude $E_{0}$ is related to $\mathcal{I}_{0}$ by $\mathcal{I}_{0}=\epsilon_{0} E_{0}^{2} c / 2$, where $\epsilon_{0}$ and $c$ are the dielectric constant and speed of light in vacuum. This leads to $E_{0}=10^{5} \mathrm{~V} / \mathrm{m}$. On the other hand, the Fermi velocity is $v_{F}=10^{6} \mathrm{~m} / \mathrm{s}$ for all $\alpha-\mathcal{T}_{3}$ materials including the dice lattice and graphene to ensure a smooth crossover to the graphene limit for $\alpha \rightarrow 0$ from an arbitrary $\alpha-\mathcal{T}_{3}$ lattice. Consequently, the interaction strength parameter $c_{0}$ is estimated as $c_{0}=e v_{F} E_{0} / \omega \backsim 10^{-22} \mathrm{~J} \backsim 0.1-1 \mathrm{meV}$. The dimensionless coupling parameter $\lambda_{0}=c_{0} /(\hbar \omega)$ will depend on two parameters, $\mathcal{I}_{0}$ and $\omega$, and both of them could be easily tuned at least by one order of magnitude so that $\lambda_{0}$ can be varied from 0.01 to nearly 1 .

We also need to bear in mind that $\lambda_{0}$ must satisfy the requirement $\lambda_{0} \ll 1$ to validate the obtained results, which are based on the off-resonant dressing-field limit. Practically, this means that we are able to accept the values of $\lambda_{0}$ in the range between 0.2 and 0.3 or, equivalently, we should limit the anisotropy factor $a_{0}\left(\lambda_{0}\right)$ around $a_{0}\left(\lambda_{0}\right)=1-\lambda_{0}^{2} \leqslant 0.9$ for graphene and $a_{0}\left(\lambda_{0}\right)=1-\lambda_{0}^{2} / 4 \leqslant 0.97$ for dice. In this paper, each left or central panel in the angular plots displays the anisotropy effect which could be achieved experimentally by applying a linearly polarized light field. The maximum possible angle for the asymmetric Klein paradox, as given by Eq. (30), could reach up to several degrees, which makes it possible for detection of this effect in an experiment or a built-in device. Quantitatively, even if we increase the value of $\lambda_{0}$ up to $\times 10$, the predicted phenomena in this paper are 


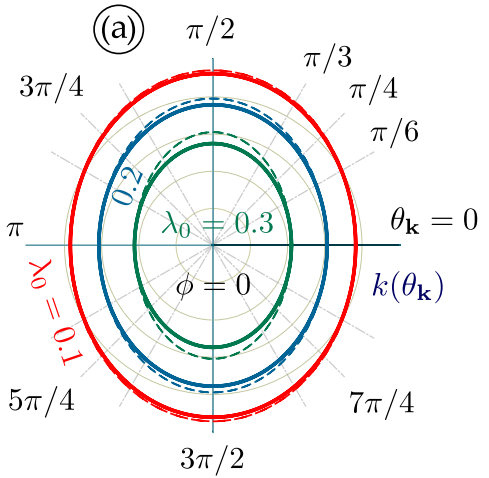

(d) $\pi / 2$

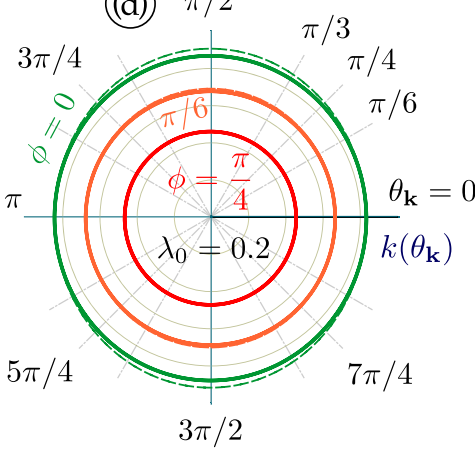

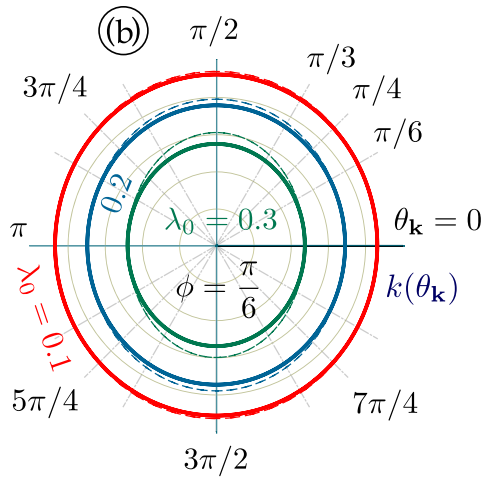

(e) $\pi / 2$

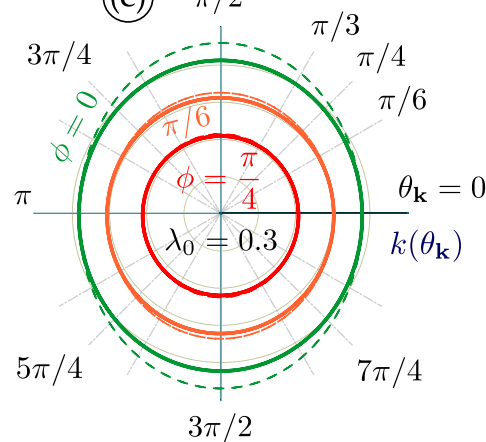

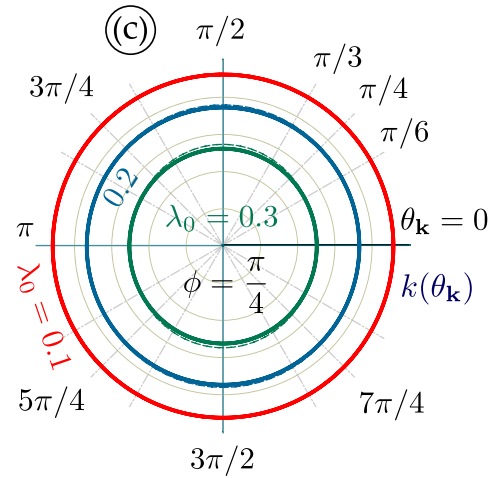

(f) $\pi / 2$

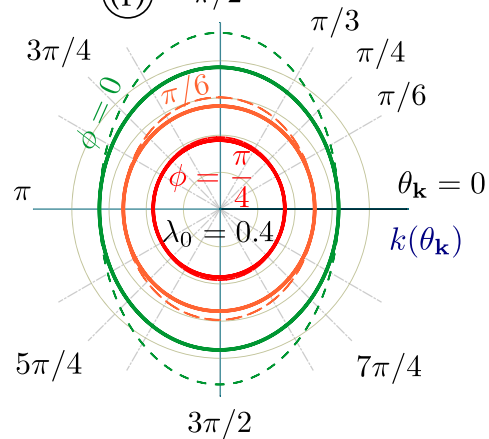

FIG. 2. Polar plots for angular dependence of $\varepsilon_{\alpha}^{\gamma= \pm 1}\left(\lambda_{0}, \boldsymbol{k}\right)$ in Eq. (15). The upper row (a)-(c) demonstrates how $\varepsilon_{\alpha}^{\gamma= \pm 1}\left(\lambda_{0}, \boldsymbol{k}\right)$ depends on the geometry phase $\phi$ with various $\lambda_{0}$ values, where curves for $\lambda_{0}=0.1$ (red), 0.2 (blue), and 0.3 (green) are displayed for $\phi=0$ (a), $\pi / 6$ (b), and $\pi / 4$ (c). Meanwhile, the lower row (d)-(f) displays $\varepsilon_{\alpha}^{\gamma= \pm 1}\left(\lambda_{0}, \boldsymbol{k}\right)$ dependence on $\lambda_{0}$ with different $\phi$ values, where curves for $\phi=0$ (green), $\pi / 6$ (orange), and $\pi / 4$ (red) are shown for $\lambda_{0}=0.2$ (d), 0.3 (e), and 0.4 (f). Here, we only present the angular dependence of each dispersion; the size of each oval is not relevant and set different for clarity. The dashed (solid) curve corresponds to the exact (expansion, up to the order of $\left.\mathcal{O}\left(\lambda_{0}^{2}\right)\right)$ calculation of $\mathcal{F}\left(\theta_{\mathbf{k}} \mid \phi, \lambda_{0}\right)$ in Eq. (16).

still valid qualitatively. However, we should not fully trust analytical expressions for anisotropy, wave functions, and transmission in this case.

In correspondence with the dressed-state energy bands in Eq. (13), their wave functions are

$$
\begin{aligned}
\Psi_{1}^{\gamma= \pm 1}\left(\tau, \lambda_{0}, \boldsymbol{k}\right) & =\frac{1}{k_{\lambda}}\left[\begin{array}{c}
k_{x}-i \tau k_{y}\left(1-\lambda_{0}^{2} / 4\right) \\
\sqrt{2} \gamma k_{\lambda} \\
k_{x}+i \tau k_{y}\left(1-\lambda_{0}^{2} / 4\right)
\end{array}\right] \\
& =\frac{1}{k_{\lambda}}\left[\begin{array}{c}
k_{x}-i \tau a_{1}\left(\lambda_{0}\right) k_{y} \\
\sqrt{2} \gamma k_{\lambda} \\
k_{x}+i \tau a_{1}\left(\lambda_{0}\right) k_{y}
\end{array}\right], \\
k_{\lambda} & =\frac{1}{\hbar v_{F}}\left|\varepsilon_{1}^{\gamma= \pm 1}\left(\lambda_{0}, \boldsymbol{k}\right)\right| \\
& =\sqrt{k_{x}^{2}+a_{1}^{2}\left(\lambda_{0}\right) k_{y}^{2}} .
\end{aligned}
$$

Equations (18) indicate that the absolute values of each component of wave function is identical, and therefore, only their phases can be changed by the dressing field. This yields

$$
\Psi_{1}^{\gamma= \pm 1}\left(\tau, \lambda_{0}, \boldsymbol{k}\right)=\frac{1}{4}\left[\begin{array}{c}
e^{-i \Theta_{\mathrm{s}}^{(1)}\left(\tau, \mathbf{k} \mid \lambda_{0}\right)} \\
\sqrt{2} \gamma \\
e^{+i \Theta_{\mathbf{S}}^{(1)}\left(\tau, \mathbf{k} \mid \lambda_{0}\right)}
\end{array}\right],
$$

where the phase factor is

$$
\begin{aligned}
\Theta_{\mathbf{S}}^{(1)}\left(\tau, \boldsymbol{k} \mid \lambda_{0}\right) & =\tan ^{-1}\left[\tau \frac{k_{y}}{k_{x}} a_{1}\left(\lambda_{0}\right)\right] \\
& =\tan ^{-1}\left[\tau a_{1}\left(\lambda_{0}\right) \tan \theta_{\mathbf{k}}\right] .
\end{aligned}
$$

The remaining wave function for the flat band is

$$
\begin{aligned}
\Psi_{1}^{\gamma=0}\left(\tau, \lambda_{0}, \boldsymbol{k}\right) & =\frac{1}{k_{\lambda}}\left[\begin{array}{c}
k_{x}-i \tau a_{1}\left(\lambda_{0}\right) k_{y} \\
0 \\
-k_{x}-i \tau a_{1}\left(\lambda_{0}\right) k_{y}
\end{array}\right] \\
& =\left[\begin{array}{c}
e^{-i \Theta_{\mathbf{S}}^{(1)}\left(\tau, \mathbf{k} \mid \lambda_{0}\right)} \\
0 \\
-e^{+i \Theta_{\mathbf{S}}^{(1)}\left(\tau, \mathbf{k} \mid \lambda_{0}\right)}
\end{array}\right] .
\end{aligned}
$$

Here, all the nonzero wave function components in Eqs. (18) and (21) have the same absolute value and differ only by a phase factor given by Eq. (20), which is not equal to $\theta_{\mathbf{k}}$ if $\lambda_{0} \neq$ 0 and depends on the light intensity, frequency, and electronlight coupling.

We also recall the results from Ref. [60] for graphene ( $\alpha=$ $0)$ under linearly polarized irradiation. The calculated energydispersion relations are $\varepsilon_{0}^{\gamma=0}\left(\lambda_{0}, \mathbf{k}\right) \equiv 0$ and

$$
\begin{aligned}
\varepsilon_{0}^{\gamma= \pm 1}\left(\lambda_{0}, \boldsymbol{k}\right) & =\gamma \hbar v_{F} k f_{\theta}\left(\lambda_{0}\right), \\
f_{\theta}\left(\lambda_{0}\right) & =\sqrt{\cos ^{2} \theta_{\mathbf{k}}+J_{0}^{2}\left(2 \lambda_{0}\right) \sin ^{2} \theta_{\mathbf{k}}} .
\end{aligned}
$$



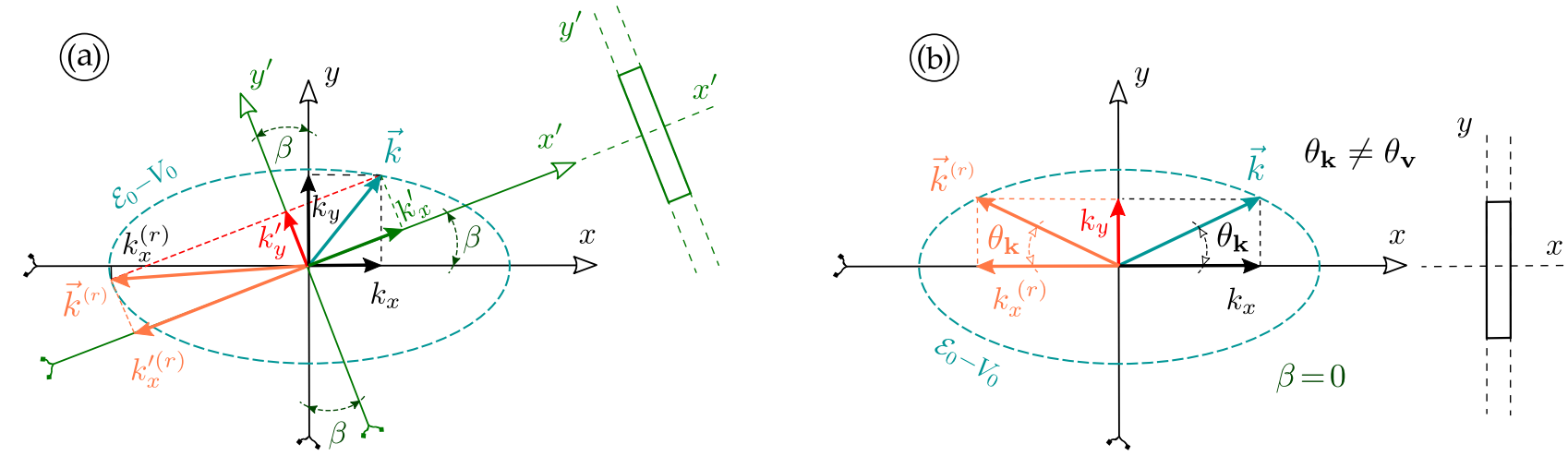

FIG. 3. Schematics for the components of wave vector $\boldsymbol{k}$ and anisotropic energy dispersions of $\alpha-\mathcal{T}_{3}$ lattices under linearly polarized irradiation. Two frames $\{x, y\}$ and $\left\{x^{\prime}, y^{\prime}\right\}$ are associated with the long axis $\hat{\boldsymbol{x}}$ of elliptical energy dispersion and the normal direction $\hat{\boldsymbol{x}}^{\prime}$ of potential barrier, respectively. These two frames are connected to each other by an in-plane rotation angle $\beta$. Here, $\boldsymbol{V}_{G}$ and $\boldsymbol{k}$ are generally not aligned $\left(\theta_{\mathbf{k}} \neq \Theta_{\mathbf{V}}\right)$ and the panels (a), (b) correspond to $\beta \neq 0$ and $\beta=0$.

Since the anisotropy factor $a_{0}\left(\lambda_{0}\right)$ can be found from the relation $\varepsilon_{0}^{\gamma= \pm 1}\left(\lambda_{0}, \boldsymbol{k}\right)=\gamma \hbar v_{F} \sqrt{k_{x}^{2}+\left[a_{0}\left(\lambda_{0}\right) k_{y}\right]^{2}}$, this gives rise to $a_{0}\left(\lambda_{0}\right)=J_{0}\left(2 \lambda_{0}\right) \approx 1-\lambda_{0}^{2}+\frac{\lambda_{0}^{4}}{4}+\cdots$, which matches Eq. (17) for a general $\alpha-\mathcal{T}_{3}$ lattice in the graphene limit $\phi \rightarrow 0$ and is expected to play a crucial role in the calculation of transmission of dressed electrons in graphene.

Moreover, the wave functions associated with Eqs. (22) for valence and conduction electrons are

$$
\begin{aligned}
\Psi_{0}^{\gamma= \pm 1}\left(\lambda_{0}, \boldsymbol{k}\right) & =\frac{1}{\sqrt{2}}\left[\begin{array}{c}
1 \\
\gamma \exp \left[i \Theta_{\mathbf{S}}^{(0)}\left(\boldsymbol{k} \mid \lambda_{0}\right)\right]
\end{array}\right], \\
\Theta_{\mathbf{S}}^{(0)}\left(\boldsymbol{k} \mid \lambda_{0}\right) & =2 \tan ^{-1}\left[\frac{\sin \theta_{\mathbf{k}} J_{0}\left(2 \lambda_{0}\right)}{\cos \theta_{\mathbf{k}}+f_{\theta}}\right] \\
& \simeq \theta_{\mathbf{k}}-\frac{\lambda_{0}^{2}}{2} \sin \left(2 \theta_{\mathbf{k}}\right)+\cdots .
\end{aligned}
$$

The wave functions in Eq. (23) are somewhat similar to those in Eq. (19) for a dice lattice. Here, two wave-function components in Eq. (23) have the same magnitude. A simple phase relation $\tan \left[\Theta_{\mathbf{S}}^{(\alpha)}\left(\boldsymbol{k} \mid \lambda_{0}\right)\right] / \tan \theta_{\mathbf{k}}=a_{\alpha}\left(\lambda_{0}\right)$ becomes a correct description for all photon-dressed $\alpha-\mathcal{T}_{3}$ materials under linearly polarized irradiation up to the order of $\backsim \lambda_{0}^{2}$, including two opposite limits for graphene and dice lattice. However, an important difference for graphene is that its wave functions have no explicit dependence on the valley index $\tau= \pm 1$.

Generally speaking, the boundary conditions for a dice lattice with $V(x) \neq 0$, as discussed in the next Sec. III, depend substantially on $k_{x}$ terms which become discontinuous at two edge boundaries of a potential barrier region. If the polarization direction of the incident light is not aligned with the $y$ axis, such a discontinuity appears in the dressed-state Hamiltonian, as demonstrated in Appendix B. Therefore, the wave-function boundary condition for a dice lattice must be modified if anisotropy exists in its energy dispersion.

\section{ASYMMETRIC ELECTRON TUNNELING IN IRRADIATED GRAPHENE AND DICE LATTICES}

We now employ the calculated energy-dispersion relation under linearly polarized irradiation for graphene and dice lattices with $V(x)=0$, as well as their wave functions in Sec. II, to study the electron transmission dynamics through a square potential barrier. The main focus stays on how to perform these calculations in the presence of an applied dressing field.

We first consider electron tunneling in irradiated graphene with the anisotropic dispersion and wave function given by Eqs. (22) and (23). To some extent, the model system is similar to the asymmetrical tunneling in multilayer phosphorene with anisotropic dispersion [71], where complete Klein transmission was obtained at a finite incident angle. However, such a system possesses some unique distinctions since the anisotropy factor of irradiated graphene relies on the intensity of imposed light (i.e., the electron-light coupling $\lambda_{0}$ ) and therefore could be tuned within the off-resonance limit $0<\lambda_{0} \ll 1$.

In addition, the polarization direction of the incident light could also be varied, instead of being parallel to that of a headon electron collision (perpendicular to the potential barrier), which is similar to a rotation of the phosphorene crystal long axis away from the normal direction of a potential barrier in Ref. [71]. The detailed formalism for the asymmetric Klein tunneling is presented in Appendix C. One of the important consequences of finite anisotropy in Dirac dispersions is that not only the magnitudes but also the directions of group velocity $\boldsymbol{V}_{G}$, spinor vector $\boldsymbol{S}$, and wave vector $\boldsymbol{k}$ are different from each other.

As we mentioned above, one can arbitrarily choose the direction for linear polarization of a dressing field, or the direction $\hat{\boldsymbol{x}}$ of long-axis for the elliptical energy dispersions in Eqs. (22), so it is not aligned with the direction $\hat{\boldsymbol{x}}^{\prime}$ for electron normal incidence on the barrier. Therefore, it is easy to introduce two nonequivalent coordinate frames $\{x, y\}$ and $\left\{x^{\prime}, y^{\prime}\right\}$, corresponding, respectively, to $\hat{\boldsymbol{x}}$ and $\hat{\boldsymbol{x}}^{\prime}$ vectors, as shown in Fig. 3. The components of a vector, such as the electron wave vector $\boldsymbol{k}$, in each of these two frames are connected by the relation in Eq. (C4), which holds true for incident and reflected waves in both barrier and zero potential regions.

All wave functions, including the incoming, reflected (with amplitudes $b$ and $r$ ), and transmitted (with amplitudes $a$ and $t$ ) waves, could be written out explicitly in three separate regions according to the schematics in Fig. 1. For region 1, 
we have

$$
\begin{aligned}
\Psi_{\gamma}^{(1)}\left(\lambda_{0}, \boldsymbol{k}\right)= & \frac{1}{\sqrt{2}} \exp \left(i k_{x^{\prime}}^{(1)} x^{\prime}\right) \exp \left(i k_{y^{\prime}} y^{\prime}\right)\left[\begin{array}{c}
1 \\
\gamma e^{i \Theta_{\mathrm{s}}^{(1)}}
\end{array}\right] \\
& +\frac{r}{\sqrt{2}} \exp \left(i k_{x^{\prime}}^{(1, r)} x^{\prime}\right) \exp \left(i k_{y^{\prime}} y^{\prime}\right)\left[\begin{array}{c}
1 \\
\gamma e^{i \Theta_{\mathbf{s}}^{(1, r)}}
\end{array}\right] .
\end{aligned}
$$

For region 2 within the barrier, we get

$$
\begin{aligned}
\Psi_{\gamma^{\prime}}^{(2)}\left(\lambda_{0}, \boldsymbol{k}\right)= & \frac{a}{\sqrt{2}} \exp \left(i k_{x^{\prime}}^{(2)} x^{\prime}\right) \exp \left(i k_{y^{\prime}} y^{\prime}\right)\left[\begin{array}{c}
1 \\
\gamma^{\prime} e^{i \Theta_{\mathrm{s}}^{(2)}}
\end{array}\right] \\
& +\frac{b}{\sqrt{2}} \exp \left(i k_{x^{\prime}}^{(2, r)} x^{\prime}\right) \exp \left(i k_{y^{\prime}} y^{\prime}\right)\left[\begin{array}{c}
1 \\
\gamma^{\prime} e^{i \Theta_{\mathrm{s}}^{(2, r)}}
\end{array}\right] .
\end{aligned}
$$

Finally, the wave function in region 3 is found to be

$$
\Psi_{\gamma}^{(3)}\left(\lambda_{0}, \boldsymbol{k}\right)=\frac{t}{\sqrt{2}} \exp \left(i k_{x^{\prime}}^{(1)} x^{\prime}\right) \exp \left(i k_{y^{\prime}} y^{\prime}\right)\left[\begin{array}{c}
1 \\
\gamma e^{i \Theta_{\mathbf{s}}^{(1)}}
\end{array}\right] .
$$

In Eqs. (24)-(26), the superscripts 1 and 2 denote the wave numbers and angles in the regions with $V_{B}=0$ and $V_{B}>0$, respectively. Additionally, the superscript $r$ is used for denoting a backward-reflected wave in contrast to the incoming and transmitted forward-going waves.

We emphasize that all the wave vector components $k_{x^{\prime}}$ and $k_{y^{\prime}}$ are given in the $\left\{x^{\prime}, y^{\prime}\right\}$ frame since they are related to the direction of incoming electrons, while the spinor angle $\Theta_{\mathbf{S}}$ and the group velocity angle $\Theta_{\mathbf{V}}$ must be defined in the $\{x, y\}$ frame and correspond to two axes of elliptical energy dispersions in Eqs. (22). In other words, we face a situation where different quantities from individual wave functions in Eqs. (24)-(26) are defined in two nonequivalent coordinate systems $\{x, y\}$ and $\left\{x^{\prime}, y^{\prime}\right\}$, which makes whole mathematical operations much more complicated.

The four undetermined amplitudes $a, b, r$, and $t$ in Eqs. (24)-(26) can be calculated from the proper boundary conditions at $x^{\prime}=0$ and $x^{\prime}=W_{B}$. We have derived these matching conditions in Appendix D by integrating all components of the eigenvalue equation for a specific Hamiltonian over a small interval $\left(-\delta x^{\prime}, \delta x^{\prime}\right)$ and then taking the limit of $\delta x^{\prime} \rightarrow 0$ afterward.

For graphene with anisotropic dispersion relations in Eqs. (22), the boundary conditions are obtained in the same way as that for the isotropic case, and therefore we only match each of two components for the wave-function spinor at both edges of region 2 [71]. This leads to the following four equations:

$$
\begin{aligned}
1+r & =a+b, \\
e^{i \Theta_{\mathrm{s}}^{(1)}}+r e^{i \Theta_{\mathrm{s}}^{(1, r)}} & =s\left(a e^{i \Theta_{\mathrm{s}}^{(2)}}+b e^{i \Theta_{\mathrm{s}}^{(2, r)}}\right), \\
a e^{i k_{x^{\prime}}^{(2)} W_{B}}+b e^{i k_{x^{\prime}}^{(2, r)} W_{B}} & =s t e^{i k_{x^{\prime}}^{(1)} W_{B}}, \\
a e^{i \Theta_{\mathrm{s}}^{(2)}+i k_{x^{\prime}}^{(2)} W_{B}}+b e^{i \Theta_{\mathrm{s}}^{(2, r)}+i k_{x^{\prime}}^{(2, r)} W_{B}} & =s t e^{i \Theta_{\mathrm{s}}^{(1)}+i k_{x^{\prime}}^{(1)} W_{B}},
\end{aligned}
$$

where $s=\gamma \gamma^{\prime}=\operatorname{sgn}\left(\mathcal{E}_{0}\right) \operatorname{sgn}\left(\mathcal{E}_{0}-V_{B}\right)= \pm 1$ is the composite index characterizing the same or different electron-hole transitions at two edges, and $\operatorname{sgn}(x)=1$ or -1 for $x>0$ or $x<0$. Furthermore, the transmission and reflection coefficients are obtained as $T\left(\mathcal{E}_{0}, \Theta_{V}^{\prime(1)} \mid \beta\right)=\left|t\left(\mathcal{E}_{0}, \Theta_{V}^{\prime(1)} \mid \beta\right)\right|^{2}$ and $R\left(\mathcal{E}_{0}, \Theta_{V}^{\prime(1)} \mid \beta\right)=\left|r\left(\mathcal{E}_{0}, \Theta_{V}^{\prime(1)} \mid \beta\right)\right|^{2}$, respectively, satisfying the relation $R\left(\mathcal{E}_{0}, \Theta_{V}^{\prime(1)} \mid \beta\right)=1-T\left(\mathcal{E}_{0}, \Theta_{V}^{\prime(1)} \mid \beta\right)$ for the conservation of electron number.
To solve the four boundary equations in Eqs. (27), we must know in advance the spinor angles for incoming and reflected waves, both outside $\Theta_{\mathbf{S}}^{(1)}, \Theta_{\mathbf{S}}^{(1, r)}$ and inside $\Theta_{\mathbf{S}}^{(2)}, \Theta_{\mathbf{S}}^{(2, r)}$ the square-barrier region. These spinor angles are determined from $k_{x}$ and $k_{y}$ components defined in the $\{x, y\}$ reference frame, whereas the electron wave numbers $k_{x^{\prime}}^{(r)}, k_{x^{\prime}}^{(1, r)}, k_{x^{\prime}}^{(2)}$, $k_{x^{\prime}}^{(2, r)}$ and $k_{y^{\prime}}=$ const in Eqs. (27) are given in the $\left\{x^{\prime}, y^{\prime}\right\}$ frame. The calculations of these seven unknowns based on initially given parameters, e.g., kinetic energy $\mathcal{E}_{0}$ of incoming particle, the height $V_{B}$ of potential barrier, and the angle $\Theta_{V}^{\prime(1)}$ between its group velocity vector and $x^{\prime}$ axis are provided in Appendix C.

The next crucial issue in our calculations is the selection rule which allows for distinguishing the transmitted and reflected waves within the barrier region. It is important to emphasize that it is the group velocity component $V_{G, x^{\prime}}^{\gamma}$ or its angle $\Theta_{V}^{\prime(1)}$ within the $\left\{x^{\prime}, y^{\prime}\right\}$ reference frame that determines the direction of a moving wave. As demonstrated in Fig. 4, only the $x^{\prime}$ component $V_{G, x^{\prime}}^{\gamma}\left(\lambda_{0}, \boldsymbol{k} \mid \beta\right)$ of the group velocity vector acquires two opposite $( \pm)$ solutions in contrast to similar components of the wave vector $\boldsymbol{k}$ or the spinor vector $\boldsymbol{S}\left(\lambda_{0}, \boldsymbol{k} \mid \beta\right)$. Explicitly, these two solutions are found to be

$$
\begin{aligned}
V_{G, x^{\prime}}^{\gamma}\left(\lambda_{0}, \boldsymbol{k} \mid \beta\right)= & \pm \gamma \frac{v_{F}}{\sqrt{2}}\left\{1+a_{0}^{2}\left(\lambda_{0}\right)+\left[1-a_{0}^{2}\left(\lambda_{0}\right)\right]\right. \\
& \left.\times \cos (2 \beta)-2\left[\frac{\hbar v_{F}}{\mathcal{E}_{0}} a_{0}\left(\lambda_{0}\right) k_{y^{\prime}}\right]^{2}\right\}^{1 / 2} .
\end{aligned}
$$

According to Ref. [71], the Klein tunneling with the perfect transmission is achieved if $\boldsymbol{k}=\{k \cos \beta, k \sin \beta\}$ or $\beta=\theta_{\mathbf{k}}$ $\left(\boldsymbol{k} \| \hat{\boldsymbol{x}}^{\prime}\right)$, which implies that $\theta_{\mathbf{k}}^{\prime}=\theta_{\mathbf{k}}-\beta=0$. In this case, however, the angle of incidence $\Theta_{V}^{\prime(1)}$ for perfect transmission is not equal to $-\beta$ as expected, but is determined by

$$
\begin{aligned}
\Theta_{V}^{\prime(1)} & =\Theta_{V}^{(1)}-\beta=\tan ^{-1}\left[a_{0}^{2}\left(\lambda_{0}\right) \tan \theta_{\mathbf{k}}\right]-\beta \\
& =\tan ^{-1}\left[a_{0}^{2}\left(\lambda_{0}\right) \tan \beta\right]-\beta .
\end{aligned}
$$

Therefore, $\Theta_{V}^{\prime(1) \max }$ in Eq. (29) can be reached when $\beta=$ $\tan ^{-1}\left[1 / a_{0}\left(\lambda_{0}\right)\right]$, leading to

$$
\begin{aligned}
\Theta_{V}^{\prime(1) \max } & =\tan ^{-1}\left(1-\lambda_{0}^{2}\right)-\tan ^{-1}\left(\frac{1}{1-\lambda_{0}^{2}}\right) \\
& \simeq-\lambda_{0}^{2}-\frac{\lambda_{0}^{4}}{2}-\frac{\lambda_{0}^{6}}{6}+\cdots,
\end{aligned}
$$

which is valid for both graphene and dice lattice.

We now turn our attention to electron tunneling in an irradiated dice lattice with the anisotropic dispersion given by Eq. (13). We first note that the geometry of Dirac cones, both isotropic and anisotropic, are exactly the same for graphene and dice lattice apart from the existence of a flat band. Therefore, all the reasoning and derivations for electron wave vectors, spinor, and group velocity angles are also applicable for dice lattice, including Eqs. (C1) and (C5) as well as a similar expression for the barrier region. We will not use the flat-band wave function to avoid the situation with zero kinetic energy in all three regions because of its infinite degeneracy for electron wave numbers. 

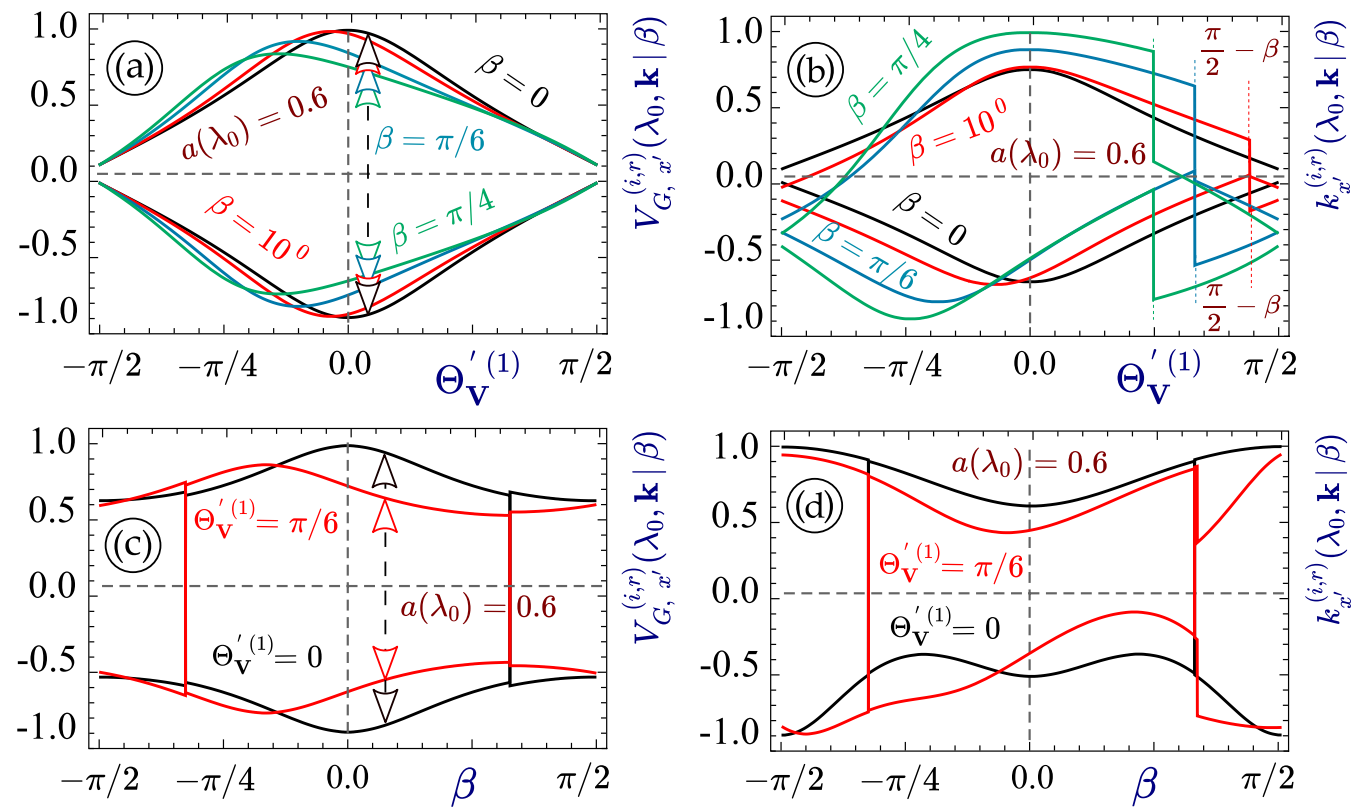

FIG. 4. $V_{G, x^{\prime}}^{\gamma}\left(\lambda_{0}, \boldsymbol{k} \mid \beta\right)\left[(\mathrm{a})\right.$, (c)] from Eq. (28) and $k_{x^{\prime}}^{(1)}[(\mathrm{b}),(\mathrm{d})]$ solved from Eq. (C5) for $a_{0}\left(\lambda_{0}\right)=0.6$ as a function of the incidence angle $\Theta_{V}^{\prime(1)}[(\mathrm{a}),(\mathrm{b})]$ and a function of rotation angle $\beta[(\mathrm{c}),(\mathrm{d})]$. In panels (a) and (c), the upper four curves correspond to the incident wave, while the lower four are for the reflected one. Two $V_{G, x^{\prime}}^{\gamma}\left(\lambda_{0}, \boldsymbol{k} \mid \beta\right)$ components for the incident and reflected waves are always with opposite signs, which is, however, not true for two $k_{x^{\prime}}^{(1)}$ components. In panels (a), (b), different curves correspond to $\beta=0,10^{\circ}, \pi / 6$ and $\pi / 4$, while $\Theta_{V}^{\prime(1)}=0$ and $\pi / 6$ are taken for two curves in plots (c), (d).

For a dice lattice, we want to find wave functions in all three regions shown in Fig. 1. Specifically, in region 1 we have

$$
\begin{aligned}
\Psi_{\gamma}^{(1)}\left(\lambda_{0}, \boldsymbol{k}\right)= & \frac{1}{4} \exp \left(i k_{x^{\prime}}^{(1)} x^{\prime}\right) \exp \left(i k_{y^{\prime}} y^{\prime}\right)\left[\begin{array}{c}
e^{-i \Theta_{\mathrm{s}}^{(1)}} \\
\sqrt{2} \gamma \\
e^{i \Theta_{\mathrm{s}}^{(1)}}
\end{array}\right] \\
& +\frac{r}{4} \exp \left(i k_{x^{\prime}}^{(1, r)} x^{\prime}\right) \exp \left(i k_{y^{\prime}} y^{\prime}\right)\left[\begin{array}{c}
e^{-i \Theta_{\mathrm{s}}^{(1, r)}} \\
\sqrt{2} \gamma \\
e^{i \Theta_{\mathrm{s}}^{(1, r)}}
\end{array}\right],
\end{aligned}
$$

where $\gamma=+1$ for electrons and -1 for holes and the spinor angle $\Theta_{\mathbf{S}}\left(\boldsymbol{k} \mid \lambda_{0}\right)$ has been presented in Eq. (20). Similarly, we find the wave function in region 2 as

$$
\begin{aligned}
\Psi_{\gamma^{\prime}}^{(2)}\left(\lambda_{0}, \boldsymbol{k}\right)= & \frac{a}{4} \exp \left(i k_{x^{\prime}}^{(2)} x^{\prime}\right) \exp \left(i k_{y^{\prime}} y^{\prime}\right)\left[\begin{array}{c}
e^{-i \Theta_{\mathrm{s}}^{(2)}} \\
\sqrt{2} \gamma^{\prime} \\
e^{i \Theta_{\mathbf{s}}^{(2)}}
\end{array}\right] \\
& +\frac{b}{4} \exp \left(i k_{x^{\prime}}^{(2, r)} x^{\prime}\right) \exp \left(i k_{y^{\prime}} y^{\prime}\right)\left[\begin{array}{c}
e^{-i \Theta_{\mathrm{s}}^{(2, r)}} \\
\sqrt{2} \gamma^{\prime} \\
e^{i \Theta_{\mathrm{s}}^{(2, r)}}
\end{array}\right],
\end{aligned}
$$

and the wave function in region 3 to be

$$
\Psi_{\gamma}^{(3)}\left(\lambda_{0}, \boldsymbol{k}\right)=\frac{t}{4} \exp \left(i k_{x^{\prime}}^{(1)} x^{\prime}\right) \exp \left(i k_{y^{\prime}} y^{\prime}\right)\left[\begin{array}{c}
e^{-i \Theta_{\mathrm{s}}^{(1)}} \\
\sqrt{2} \gamma \\
e^{i \Theta_{\mathrm{s}}^{(1)}}
\end{array}\right] .
$$

It is straightforward to verify that for the case of an isotropic dispersion with $a_{1}\left(\lambda_{0}\right)=1$ and $\beta=0$, the spinor angle $\Theta_{\mathbf{S}}$ is the same as the wave-vector angle $\theta_{\mathbf{k}}$. Meanwhile, we also acquire $\Theta_{\mathbf{S}}^{(1, r)} \rightarrow \pi-\theta_{\mathbf{k}}^{(1)}$ and $\Theta_{\mathbf{S}}^{(2, r)} \rightarrow \pi-\theta_{\mathbf{k}}^{(2)}$ in this case. Furthermore, the electron wave numbers for the forward and backward waves become $k_{x^{\prime}}^{(1, r)} \rightarrow-k_{x}^{(1)}, k_{x^{\prime}}^{(2, r)} \rightarrow$ $-k_{x}^{(2)}, k_{x^{\prime}}^{(1)} \rightarrow k_{x}^{(1)}$, and $k_{y^{\prime}} \rightarrow k_{y}$. Finally, Eq. (C5) gives rise to $k_{x}^{(1)} \rightarrow \sqrt{\left(\mathcal{E}_{0} / \hbar v_{F}\right)^{2}-k_{y}^{2}}$ with the conserved wave number $k_{y}$ in scattering events. In this way, all previously obtained expressions for the electron tunneling in graphene $[3,46]$ can be transformed into corresponding ones for dice lattice [43].

However, the boundary conditions for an anisotropic dice lattice, as derived in Appendix D, are different from those for graphene (i.e., we cannot simply match individual components of a wave function) or even not equivalent to those for an isotropic dice lattice since the additional $k_{x^{\prime}}$-dependent term in the dressed-state Hamiltonian leads to additional instances of discontinuity at the boundaries of a barrier region. The new composite boundary conditions are

$$
\varphi_{2}\left(-\delta x^{\prime}\right)=\varphi_{2}\left(\delta x^{\prime}\right)
$$

and

$$
\begin{aligned}
& c_{\tau}^{+}\left(\lambda_{0}, \beta\right) \varphi_{1}\left(-\delta x^{\prime}\right)+c_{\tau}^{-}\left(\lambda_{0}, \beta\right) \varphi_{3}\left(-\delta x^{\prime}\right) \\
& \quad=c_{\tau}^{+}\left(\lambda_{0}, \beta\right) \varphi_{1}\left(\delta x^{\prime}\right)+c_{\tau}^{-}\left(\lambda_{0}, \beta\right) \varphi_{3}\left(\delta x^{\prime}\right),
\end{aligned}
$$

where $c_{\tau}^{ \pm}\left(\lambda_{0}, \beta\right)=\tau \cos \beta \pm i a_{1}\left(\lambda_{0}\right) \sin \beta$.

The new boundary conditions in Eq. (35) for a dice lattice with anisotropic Dirac cone dispersion represent one of the key results of the present paper. Consequently, the equations derived from the above boundary conditions can be utilized to determine the transmission and reflections amplitudes with 
explicit equations given by

$$
\begin{aligned}
& 1+r=s(a+b), \\
& \cos \left[\Theta_{\mathbf{S}}^{(1)}-\alpha_{\tau}\left(\beta, a_{1}\right)\right]+r \cos \left[\Theta_{\mathbf{S}}^{(r, 1)}-\alpha_{\tau}\left(\beta, a_{1}\right)\right] \\
& =a \cos \left[\Theta_{\mathbf{S}}^{(2)}-\alpha_{\tau}\left(\beta, a_{1}\right)\right]+b \cos \left[\Theta_{\mathbf{S}}^{(r, 2)}-\alpha_{\tau}\left(\beta, a_{1}\right)\right] \text {, } \\
& a \exp \left(i k_{x^{\prime}}^{(2)} W_{B}\right)+b \exp \left(i k_{x^{\prime}}^{(2, r)} W_{B}\right)=t s \exp \left(i k_{x^{\prime}}^{(1)} W_{B}\right) \text {, } \\
& a \cos \left[\Theta_{\mathbf{S}}^{(2)}-\alpha_{\tau}\left(\beta, a_{1}\right)\right] \exp \left(i k_{x^{\prime}}^{(2)} W_{B}\right) \\
& +b \cos \left[\Theta_{\mathrm{S}}^{(r, 2)}-\alpha_{\tau}\left(\beta, a_{1}\right)\right] \exp \left(i k_{x^{\prime}}^{(2, r)} W_{B}\right) \\
& =t s \cos \left[\Theta_{\mathrm{S}}^{(1)}-\alpha_{\tau}\left(\beta, a_{1}\right)\right] \exp \left(i k_{x^{\prime}}^{(1)} W_{B}\right) \text {, }
\end{aligned}
$$

where $\alpha_{\tau}\left(\beta, a_{1}\right)=\tau \tan ^{-1}\left[a_{1}\left(\lambda_{0}\right) \tan \beta\right]$ and $s=\gamma \gamma^{\prime}=$ $\operatorname{sign}\left(\mathcal{E}_{0}\right) \operatorname{sign}\left(\mathcal{E}_{0}-V_{B}\right)= \pm 1$ is a composite index characterizing a possible electron $\rightarrow$ hole $\rightarrow$ electron transition, similarly to what we have obtained for graphene. From Eqs. (36), we know that once $\boldsymbol{k}$ and $\Theta_{\mathbf{S}}$ are known, the transmission and reflection amplitudes, $t$ and $r$, could be calculated in a straightforward way.

Although we do not consider any collective effects or Fermi surface here, it is convenient to express all quantities and their numerical values in terms of a single unit corresponding to a typical Fermi momentum $k_{F}^{(0)}$ of graphene electrons. Specifically, we set $k_{F}^{(0)}=\sqrt{2 \pi n_{0}} \backsim$ $10^{4}-10^{5} \mathrm{~cm}^{-1}$ for a standard, or rather dilute, 2D electron density $n_{0}=10^{8}-10^{10} \mathrm{~cm}^{-2}$. Therefore, we are able to measure all lengths, such as barrier widths, in terms of $l_{0}=1 / k_{F}^{(0)} \backsim 10^{-5} \mathrm{~cm} \backsim 100 \mathrm{~nm}$, while the unit for energy can be taken as $E_{0}=\hbar v_{F} k_{F}^{(0)} \leqslant 1 \mathrm{meV}$. In fact, such a low-electron energy is required so as to satisfy the offresonant condition for the dressing field with a terahertz frequency.

\section{NUMERICAL RESULTS AND DISCUSSION}

The particular features for electron tunneling, transmission, and reflection amplitudes in a given irradiated material are mainly governed by its band structure, as well as the properties of dressed-state wave functions of electrons. For both graphene and dice lattice, linearly polarized irradiation creates anisotropy between in-plane components of the electron wave vector and modifies the phase factors of individual wavefunction components, while magnitudes for the components of wave functions remain unchanged.

Such light-induced modifications of material properties are found to be quite different for graphene and dice lattice mainly due to the presence of the flat band in the energy dispersions of the dice. However, the preserved symmetry of wave functions results in the occurrence of anomalous or asymmetric Klein paradox in both materials.

Klein paradox, a complete transmission of incoming particles independent of barrier height and width, has become one of the most important and well-studied topics in 2D Dirac cone lattices. This phenomenon is distinguished from the resonant Fabry-Perot tunneling resulting from constructive interference between incoming and reflected waves on both sides of a barrier region and is not possible for a step with only one potential discontinuity.

The transmission peaks of both types could be easily resolved and identified for the case of a standard isotropic Dirac spectrum since the Klein paradox only occurs for the head-on collision of incoming particles on a potential barrier. In fact, the following approximate expression for electron transmission through a high potential barrier $V_{B} \gg \mathcal{E}_{0}$ [3],

$$
T_{0}\left(\mathcal{E}_{0}, \theta_{\mathbf{k}}^{(1)} \mid \beta=0\right) \approx \frac{\cos ^{2} \theta_{\mathbf{k}}^{(1)}}{1-\cos ^{2}\left(k_{x}^{(2)} W_{B}\right) \sin ^{2} \theta_{\mathbf{k}}^{(1)}},
$$

suggests that the Klein paradox with complete transmission and zero reflection are always present for the head-on collision with $\theta_{\mathbf{k}}^{(1)}=0$, but there also exists a number of other resonances of unimpeded tunneling corresponding to $k_{x}^{(2)} W_{B}=$ $\pi \times$ integer with their peak locations depending on the barrier width $W_{B}$ and the longitudinal wave number $k_{x}^{(2)}$ within the barrier region. The latter quantity is determined from the relation involving the kinetic energy $\mathcal{E}_{0}$ of incoming particles and the barrier height $V_{B}$.

In the case of asymmetrical tunneling, it it difficult to determine which peak of electron transmission is associated with the Klein paradox, while all the other peaks represent different types of transmission resonances and are beyond the focus of our current investigation. For this reason, we always display transmission results with different potential barrier widths and heights in each polar plot, as presented in Figs. 5-10 for both graphene and dice lattice. In this way, the position of the anomalous Klein paradox can be unambiguously determined.

The results for transmission depend on various parameters, related to material properties of each considered lattice, strength and frequency of the applied irradiation, angle $\beta$ between the $x$ and $x^{\prime}$ axis discussed above, which could accept values within $(-\pi / 2, \pi / 2)$ range disregarding of the intensity of a dressing field, the barrier height $V_{B}$ and width $W_{B}$. Each of these quantities has a unique and rather complicated effect on electron transmission, which makes presentation in this paper quite lengthy and elaborate.

In an effort not to overload readers with many different cases and parameters, we only provide some representative examples for electron transmission with emphasis on low anisotropy $a\left(\lambda_{0}\right)$, which could be induced and tuned by external irradiation, and rather small angles $\beta$, which leads to maximized asymmetry in the Klein paradox [75].

From the perspective of practical applications, the anisotropy in electron dispersions might be tuned externally by varying the intensity of applied linearly polarized electromagnetic radiation as seen from the electron-light coupling parameter $\lambda_{0}$. Apart from that, we also change the angle $\beta$ between directions of the potential barrier (a perpendicular) and the long axis of elliptical energy dispersions, i.e., changing the linear-polarization direction of the imposed dressing field. All these factors can greatly affect the location and properties of anomalous Klein tunneling.

If the misalignment angle $\beta$ between the electron wave numbers $k_{x}$ and $k_{x^{\prime}}$ is zero, the observed Klein peak is symmetric and corresponds to the head-on collision, like in regular graphene [46] or a dice lattice [43]. However, the off-peak 

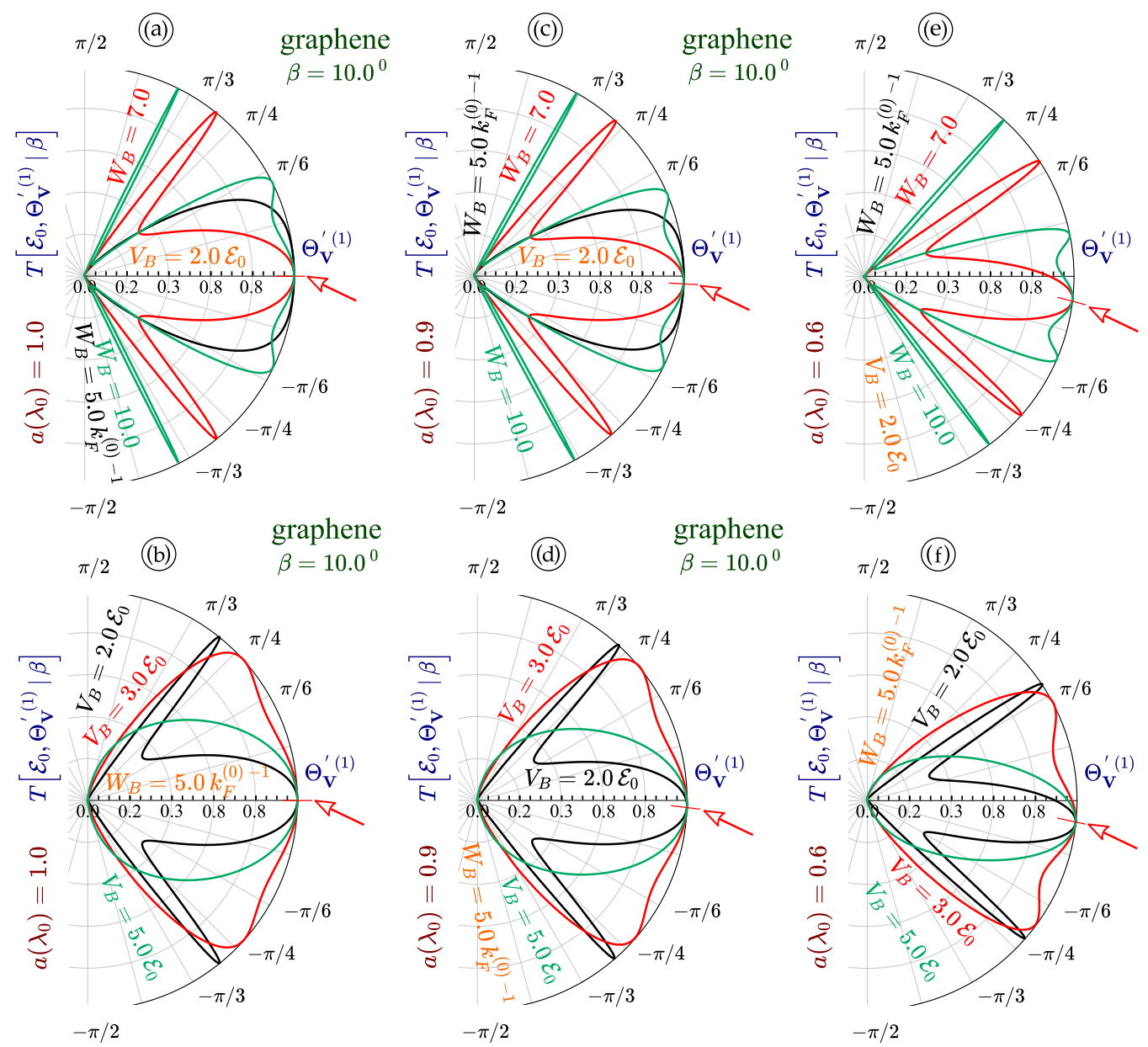

FIG. 5. Angular plots for transmission $T\left(\mathcal{E}_{0}, \Theta_{V}^{\prime(1)} \mid \beta\right)$ as a function of the group velocity direction $\Theta_{V}^{\prime(1)}$ of the incident particles in graphene. Each panel is related to a specific value of $a_{0}\left(\lambda_{0}\right)=1.0$ for (a), (b); 0.9 for (c), (d); 0.6 for (e), (f). Panels (a), (c), (e) demonstrate the transmission by black, red, and green curves for $k_{F}^{(0)} W_{B}=5,7,10$, and $V_{B} / \mathcal{E}_{0}=2$, as well as for $V_{B} / \mathcal{E}_{0}=2,3,5$ and $k_{F}^{(0)} W_{B}=5$ in $(\mathrm{b})$, (d), (f). The direction of the shifted non-head-on Klein paradox is indicated by the red arrow in each panel. Here, misalignment angle $\beta=10^{\circ}$ is set for all panels.

transmission curves are distorted toward a narrow peak with a lower transmission, as we demonstrate and explain in the Supplemental Material [75]. If angle $\beta$ is made finite, on the other hand, we will always find a shift of the Klein peak for any level of anisotropy $a\left(\lambda_{0}\right)$, and the results become rather different by comparing graphene with a dice lattice, as shown in Figs. 5-8.

First, for graphene we find that the direction of anomalous Klein paradox angle $\Theta_{V}^{\prime(1)}$ is gradually shifted downward away from the head-on collision direction with increasing $\beta$, or when the anisotropy in energy dispersions of electrons is enhanced, as can be verified from (e) and (f) of Fig. 5. Moreover, such a unique feature persists when $\beta$ is further increased, as can be seen by comparing panels (e) and (f) of Fig. 5.

Next, for dice lattices, the variation of angle $\Theta_{V}^{\prime(1)}$ with enhanced anisotropy by a reduced $a_{1}\left(\lambda_{0}\right)$ becomes less significant due to appearance of a very broad anomalousKlein-paradox peak in this case, as shown in panels (e) and (f) of Figs. 6. However, the angle deviation from $\Theta_{V}^{\prime(1)}=0$ still increases with $\beta$ for dice lattices. Moreover, the direction of anomalous-Klein-paradox peak is found fixed in various panels of Fig. 6, although the angle distributions of other resonant-tunneling peaks do change with barrier width $W_{B}$ or barrier height $V_{B}$.

Furthermore, we consider the transmission with a specific rotation angle $\beta=\tan ^{-1}\left[1 / a_{\{0,1\}}\left(\lambda_{0}\right)\right]$ in Fig. 7 for graphene and Fig. 8 for dice lattices, which leads to the biggest deviation angle between the anomalous Klein tunneling direction and the direction of head-on incidence. In this case, we find that the angle for anomalous Klein tunneling increases with decreased $a_{\{0,1\}}\left(\lambda_{0}\right)$ value, but the resulting variation becomes noticeable only for a larger anisotropy with $a_{\{0,1\}}\left(\lambda_{0}\right)=0.6$. However, we also notice that the condition for $a_{\{0,1\}}\left(\lambda_{0}\right)=0.6$ cannot be met by applying an off-resonant dressing field and, therefore, the results presented in Figs. 7 and 8 are only for the purpose of comparison.

We emphasize that the most general configuration is considered here for the square-barrier transmission of electron states with anisotropic Dirac cone dispersions. In principle, there are multiple ways to achieve such states apart from applying linearly polarized light. For example, one can use a 2D substrate with anisotropic electronic states, such as phos- 


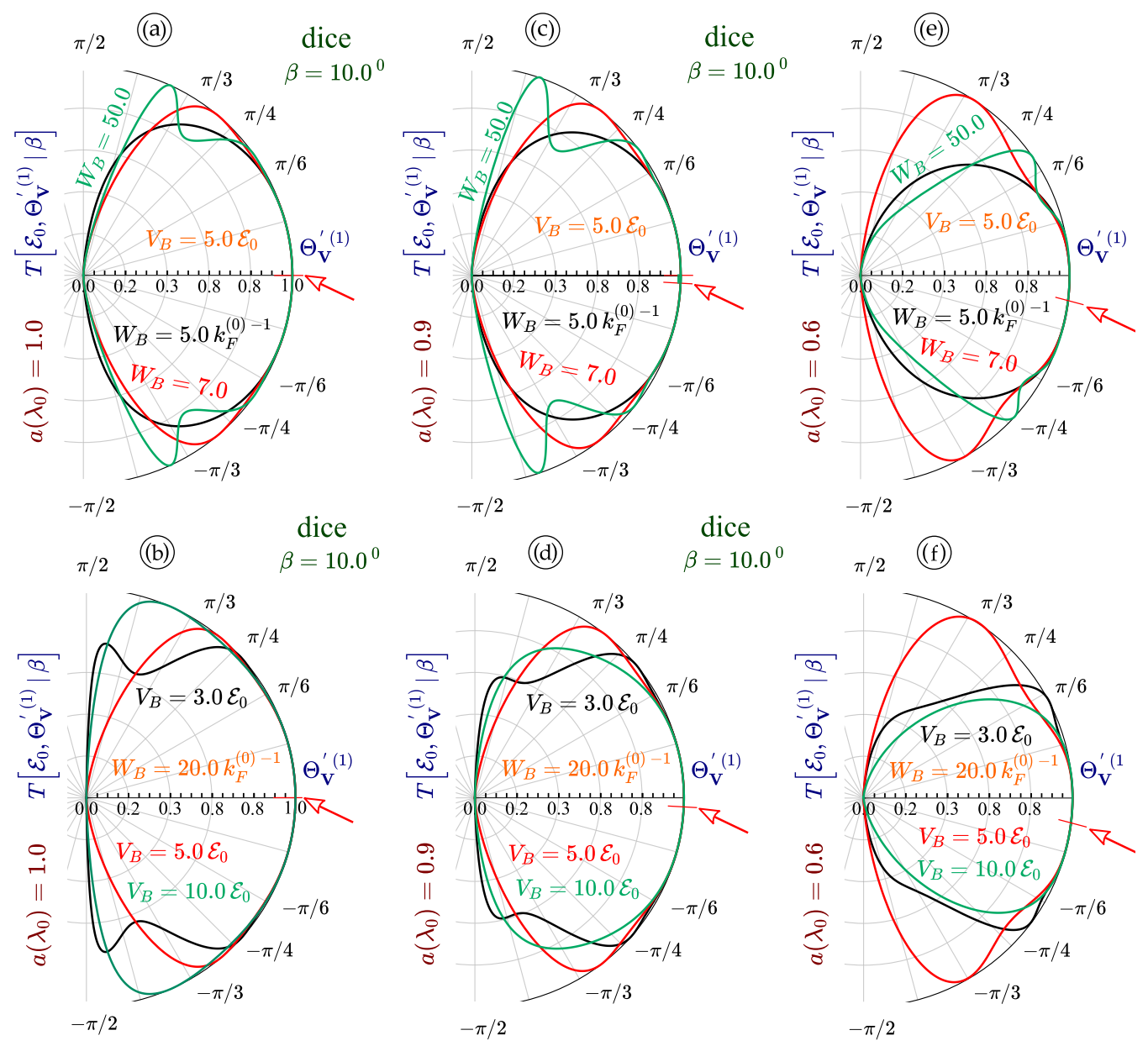

FIG. 6. Angular plots for transmission $T\left(\mathcal{E}_{0}, \Theta_{V}^{\prime(1)} \mid \beta\right)$ as a function of the group velocity direction $\Theta_{V}^{\prime(1)}$ of the incident particles in dice lattices. Each panel relates to a specific value of $a_{1}\left(\lambda_{0}\right)=1.0$ for (a), (b); 0.9 for (c), (d); 0.6 for (e), (f). Panels (a), (c), (e) demonstrate the transmission by black, red, and green curves for $k_{F}^{(0)} W_{B}=5,7,50$, and $V_{B} / \mathcal{E}_{0}=5$, as well as for $V_{B} / \mathcal{E}_{0}=3,5,10$, and $k_{F}^{(0)} W_{B}=20$ in $(\mathrm{b})$, (d), (f). The direction of the shifted non-head-on Klein paradox is indicated by the red arrow in each panel. Here, misalignment angle $\beta=10^{\circ}$ is set for all panels.

phorene, which could induce an anisotropy in the 2D layer at any desired levels. If new experimental methods become available for inducing a strong anisotropy in graphene or dice lattice, the current theory on transmission will definitely be applicable to these cases.

The dashed arrows shown in Figs. 7 and 8 mark the locations of the transmission peaks corresponding to $\beta=40^{\circ}$ (see last two figures in the Supplemental Material). In doing this, we intend to demonstrate that such a $\beta$ angle, corresponding to $\tan \beta=a\left(\lambda_{0}\right)$, results in the largest asymmetry for Klein transmission peaks and the difference in angular distribution of Klein peak is noticeable even if these two angles are $40^{\circ}$ and $48^{\circ}$ in panels (c) and (d). That is, for any values of anisotropy factor $a\left(\lambda_{0}\right)$, there exists a specific critical angle $\beta$ for the largest asymmetry of the central Klein transmission maximum.

Very importantly, we have compared the results for transmissions in graphene and dice lattice and shown some noticeable distinction between them due to the fact that one need employ new boundary conditions for a dice lattice which are different from directly matching each wave-function components, as we discussed in Sec. III. Indeed, we have found that the transmission for a dice lattice is considerably larger than that for graphene under similar conditions, as seen especially well from the density plots in Figs. 9 and 10 for a significantly expanded bright region. This happens due to the so-called magic case, i.e., a complete transmission occurs for the full range of incident angles $\Theta_{V}^{\prime(1)}$ if the incoming particle energy satisfies $\mathcal{E}_{0} / E_{F}^{(0)}=1 / 2$, which remains in place for anisotropic dispersions as well as a finite rotation angle $\beta \neq 0$.

The angular distributions of other resonant peaks (not related to Klein paradox) also vary significantly between graphene and dice lattices, in addition to widened transmission peaks of the latter. In contrast, graphene exhibits sharp satellite peaks under similar external conditions. Figures 9 and 10 for graphene and dice lattices clearly demonstrate that the angle for anomalous Klein paradox does not depend on the kinetic energy $\mathcal{E}_{0}$ of incoming particles, which is in agreement with our theoretical model.

Meanwhile, the transmission results in Fig. 8 for dice lattices do not display any dependence on the valley index $\tau= \pm 1$ even though $\tau$ appears in the boundary conditions in Eqs. (36). Such a conclusion is not expected to be the case for 

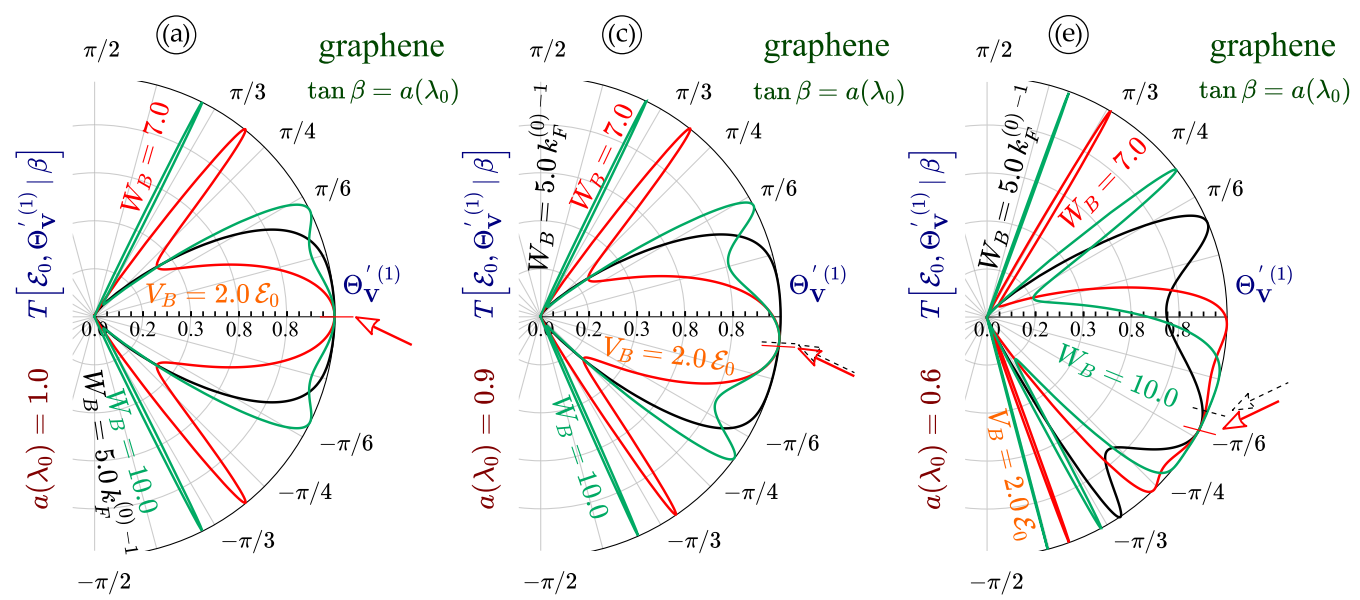

(b)) $\beta=0.785=45.0^{0}$
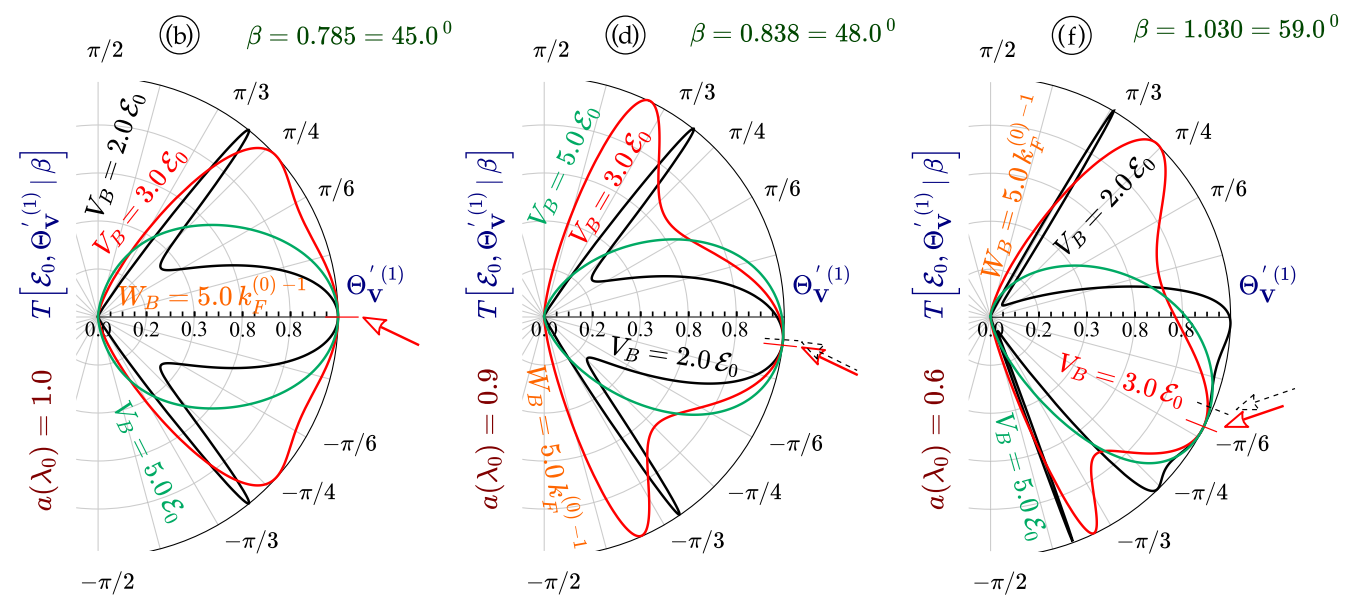

FIG. 7. Angular plots for $T\left(\mathcal{E}_{0}, \Theta_{V}^{\prime(1)} \mid \beta\right)$ as a function of $\Theta_{V}^{\prime(1)}$ in graphene. Each panel relates to a specific value of $a_{0}\left(\lambda_{0}\right)=1.0$ for (a), (b); 0.9 for (c), (d); 0.6 for (e), (f). Panels (a), (c), (e) demonstrate the transmission by black, red, and green curves for $k_{F}^{(0)} W_{B}=5,7,10$, and $V_{B} / \mathcal{E}_{0}=2$, as well as for $V_{B} / \mathcal{E}_{0}=2,3,5$ and $k_{F}^{(0)} W_{B}=5$ in (b), (d), (f). The direction of the shifted non-head-on Klein paradox is indicated by the red arrow in each panel. Here, $\tan \beta=1 / a_{0}\left(\lambda_{0}\right)$ is set for all panels, and then, $\beta=45^{\circ}, 48^{\circ}, 59^{\circ}$ correspond to (a), (b); (c), (d); (e), (f), respectively.

general $\alpha-\mathcal{T}_{3}$ lattices with more complicated dependence on index $\tau$ in its wave functions and transmissions.

As a whole, there are several independent parameters in this problem, and the effect of each of them on electron tunneling has been carefully examined. We have investigated how a nonzero angle for Klein-paradox depends on the anisotropy factor $a\left(\lambda_{0}\right)$ due to a linearly polarized dressing field, as well as on the misalignment angle $\beta$ between crystal long axis and the normal of a potential barrier. These two parameters could be independently controlled by the direction of polarization for irradiation and its intensity. For a given $a\left(\lambda_{0}\right)$, there exists a specific $\beta$ value at which the angle for Klein paradox reaches a maximum value in graphene and dice lattice, as shown in Figs. 7 and 8. The plots of numerical results for nonzero Klein-paradox angle faithfully represent the analytic expression in Eq. (30) for both graphene and dice lattice.

\section{CONCLUDING REMARKS}

In this paper, we have investigated the non-head-on, or asymmetrical, Klein tunneling in graphene and a dice lattice in the presence of a linearly polarized dressing field. Such anomalous Klein tunneling results uniquely from the misalignment of optically controlled elliptical dispersion for Dirac dressed states and the direction of incident particles. Specifically, we have performed a careful theoretical investigation on the electron tunneling across a square finite-width potential barrier in graphene, as well as in a pseudospin-1 dice model, with laser-induced anisotropic energy dispersions in their valence and conduction bands. Such a tunable anisotropy can be induced in a 2D material by applying a linearly polarized irradiation with polarization direction different from the long axis of elliptical energy dispersion of Dirac cone dressed states. Klein tunneling is really important and one of the most thoroughly investigated quantum phenomena in all recently discovered 2D Dirac materials.

The electron dynamics of optically controllable dressed states have been explored theoretically by using FloquetMagnus perturbative expansion approach for electron-light interaction Hamiltonians. In particular, we have found that the effect of high-frequency linearly polarized irradiation for both graphene and dice lattice can lead to an intensity-dependent modification to the quantum phases of dressed states, which is characterized by a spinor angle different from the incident angle of incoming particles. Here, the direction of incident particles is measured with respect to the surface normal of 


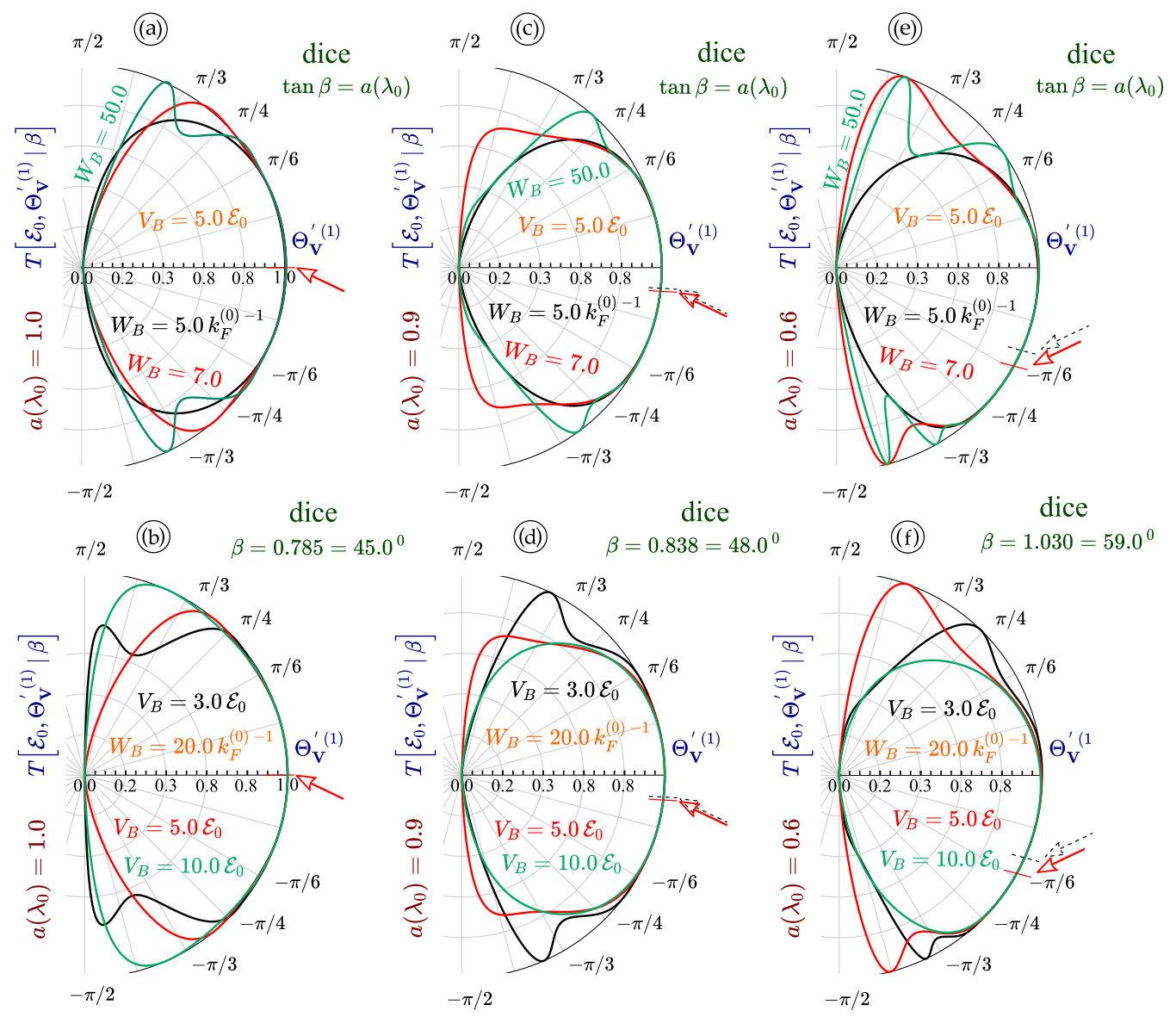

FIG. 8. Angular plots for $T\left(\mathcal{E}_{0}, \Theta_{V}^{\prime(1)} \mid \beta\right)$ as a function of $\Theta_{V}^{\prime(1)}$ in dice lattices. Each panel relates to a specific value of $a_{1}\left(\lambda_{0}\right)=1.0$ for (a), (b); 0.9 for (c), (d); 0.6 for (e), (f). Panels (a), (c), (e) demonstrate the transmission by black, red, and green curves for $k_{F}^{(0)} W_{B}=5,7,50$ and $V_{B} / \mathcal{E}_{0}=5$, as well as for $V_{B} / \mathcal{E}_{0}=3,5,10$, and $k_{F}^{(0)} W_{B}=20 \mathrm{in}(\mathrm{b})$, (d), (f). The direction of the shifted non-head-on Klein paradox is indicated by the red arrow in each panel. Here, $\tan \beta=1 / a_{1}\left(\lambda_{0}\right)$ is set for all panels, and then $\beta=45^{\circ}, 48^{\circ}, 59^{\circ}$ correspond to (a), (b); (c), (d); (e), (f), respectively.

a potential barrier, while the direction of the spinor vector of a dressed-state wave-function is quantified with respect to the semimajor axis of the elliptical energy dispersion of electrons. Therefore, two individual coordinate frames of reference must be introduced specifically for these two different directions. However, there exists an angle misalignment between these two frames due to the presence of intensitydependent anisotropy in the energy dispersion of dressed-state electrons under linearly polarized irradiation.

Making use of the unique electronic properties from these linear-polarization coupled electron dressed states in graphene and dice lattice, we have investigated their transmission and found the so-called anomalous Klein paradox with a peak in the angular distribution of transmission away from the head-on direction for incoming particles. This finite incident angle depends on the degree of anisotropic energy dispersion $a_{\{0,1\}}\left(\lambda_{0}\right)$ or the electron-light coupling constant $\lambda_{0}$, as well as the misalignment angle $\beta$ between the surface normal of a potential barrier and the semi-major axis of the elliptical energy dispersion of dressed-state electrons. Moreover, the maximum angle deviation for anomalous Klein paradox is achieved as $\beta=\tan ^{-1}\left[1 / a_{\{0,1\}}\left(\lambda_{0}\right)\right]$, somewhat similar to the case of phosphorene [71] with material-based anisotropic band structures. However, the phenomena which we have investigated are unique because both the light-induced anisotropy and the misalignment angle $\beta$ are tunable and could be varied externally.

We have calculated the $x^{\prime}$ components (perpendicular to the potential barrier) of electron wave vector, spinor vector, and group velocity in barrier regions and consequently determined that only the last quantity has two opposite solutions. Therefore, only the longitudinal component of electron group velocity should be used to distinguish different solutions corresponding to transmitted and reflected electron waves. The analytic expression for the $x^{\prime}$ component of the group velocity vectors for incoming and reflected waves could also be of special interest.

Apart from the location of the angle for anomalous Klein paradox, the angular distributions of other resonant peaks in both transmission and reflection probabilities appear to be quite different for graphene and dice lattice. Quantitatively, a dice lattice can acquire much larger off-peak transmission amplitudes compared to graphene under the same conditions, and in particular, the magic case for complete transmission covering the full range of incident angle is seen at $\mathcal{E}_{0}=V_{B} / 2$ for both graphene and dice lattices, independent of the degree of anisotropy $a_{\{0,1\}}\left(\lambda_{0}\right)$ and the value of misalignment angle $\beta$. 

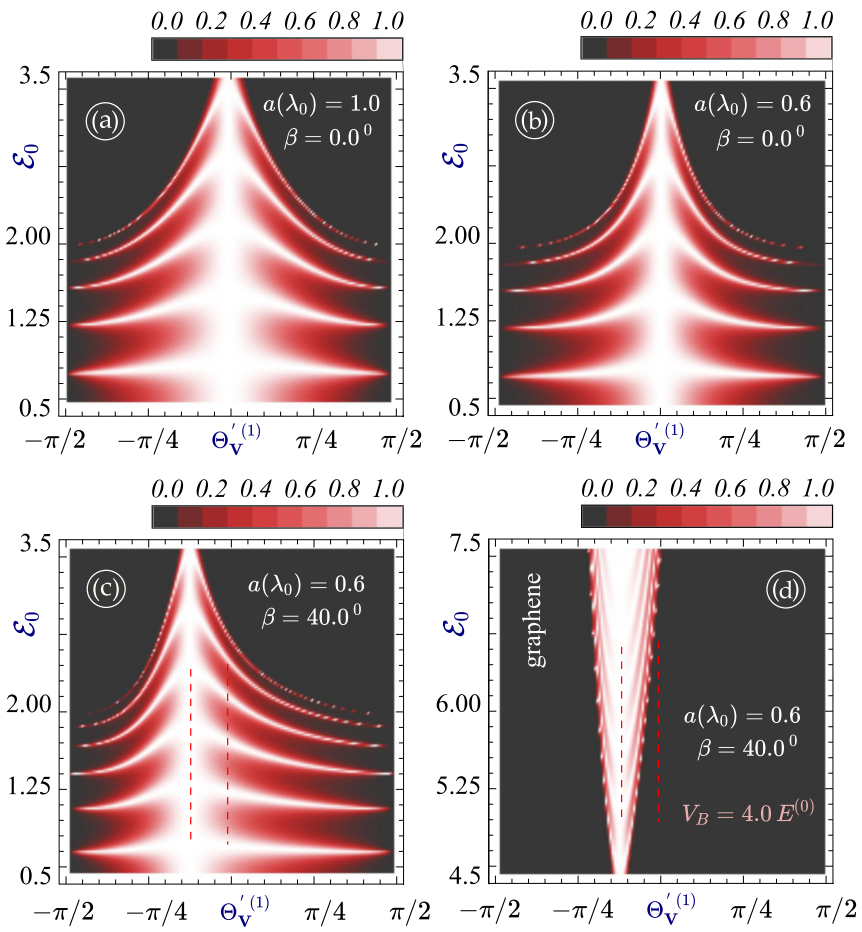

FIG. 9. Density plots for $T\left(\mathcal{E}_{0}, \Theta_{V}^{\prime(1)} \mid \beta\right)$ as functions of $\Theta_{V}^{\prime(1)}$ and $\mathcal{E}_{0} / E_{F}^{(0)}$ in graphene with $V_{B} / E_{F}^{(0)}=4$. Panel (a) is for $a_{0}\left(\lambda_{0}\right)=$ 1 , while panels (b) and (d) are for $a_{0}\left(\lambda_{0}\right)=0.6$. Upper plots (a), (b) correspond to $\beta=0^{\circ}$, but lower plots to $\beta=40^{\circ}$. Panels (c), (d) are plotted for the same values of $a_{0}\left(\lambda_{0}\right)$ and $\beta$ and, therefore, differ only by the energy range for display. Particularly, plot (d) shows the transmission for the range of $\mathcal{E}_{0}$ above the barrier height $V_{B}$.

Compared to the known boundary conditions for graphene with pseudospin-1/2 Hamiltonian in Eqs. (D6), the derived boundary conditions for a dice lattice with pseudospin-1 Hamiltonian acquire additional terms, as seen in Eq. (35). Such a modification seems counterintuitive and largely unexpected since this is never the case in studying familiar pseudospin-1/2 Dirac materials. The derivation of mixed boundary conditions in this paper could be utilized by others in matching the wave functions with anisotropic dispersions in all sorts of $\alpha-\mathcal{T}_{3}$ materials, including a dice lattice, such as an irradiated $\mathrm{SrTiO}_{3} / \mathrm{SrIrO}_{3} / \mathrm{SrTiO}_{3}$ trilayer.

For a chosen material, its low-energy Hamiltonian and the set of its lattice parameters are unique and cannot be reproduced exactly in any other lattice. This leads to specific electronic states and unique material properties addressed in this paper, such as the electronic and ballistic transport. On the other hand, the difference between a dice lattice and graphene can be attributed to the presence of a flat band in the energy spectrum of the former, and a variety of consequences from its presence as well, such as a specific form of the wave function and its components and additional electron transitions from or to this flat band.

The theoretical results could be practically implemented into an extremely wide range of recently discovered Dirac materials either with a built-in anisotropic energy dispersion or with an externally tunable anisotropy due to incident irradiation. In the absence of anisotropy in energy dispersion,
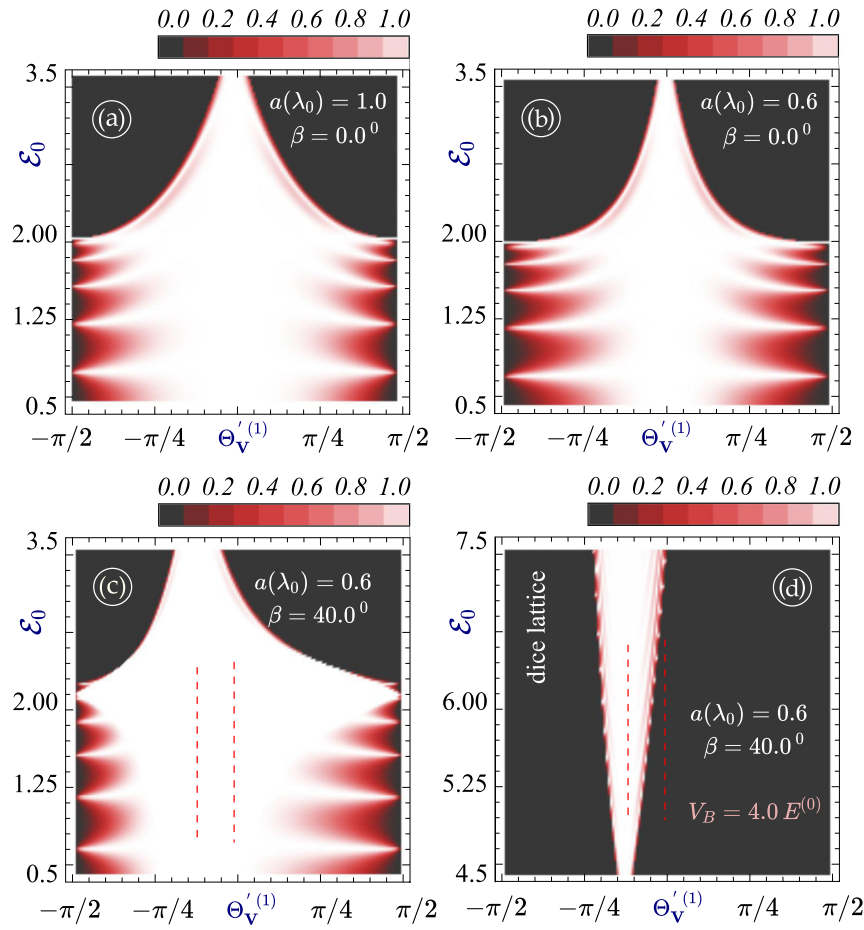

FIG. 10. Density plots for $T\left(\mathcal{E}_{0}, \Theta_{V}^{\prime(1)} \mid \beta\right)$ as a function of $\Theta_{V}^{\prime(1)}$ and $\mathcal{E}_{0} / E_{F}^{(0)}$ in dice lattices with $V_{B} / E_{F}^{(0)}=4$. Panel (a) is for $a_{1}\left(\lambda_{0}\right)=1$, while panels (b) and (d) are for $a_{1}\left(\lambda_{0}\right)=0.6$. Upper plots (a), (b) correspond to $\beta=0^{\circ}$, but lower plots to $\beta=40^{\circ}$. Panels (c), (d) are plotted for the same values of $a_{1}\left(\lambda_{0}\right)$ and $\beta$ and, therefore, differ only by the energy range for display. Particularly, plot (d) shows the transmission for the range of $\mathcal{E}_{0}$ above the barrier height $V_{B}$.

this system behaves much like $n$ - $p$ - $n$ multijunctions with an additional electric gate to control an electrically injected current by a positive base voltage for tuning barrier height $V_{B}$. In the presence of laser-tunable anisotropic energy dispersion, on the other hand, an antenna-coupled incident laser can be employed as an optical gate to control both the magnitude and direction of an injected ballistic current through the angle-dependent electron transmission $T\left(\mathcal{E}_{0}, \Theta_{V}^{\prime(1)} \mid \beta\right)$. The closed-form analytic expressions obtained in this paper are the most valuable and desirable for researchers in the fields of low-dimensional condensed-matter physics and optoelectronics, especially for experimentalists. Undoubtedly, the electronic properties of both coherent tunneling and ballistic transport of electrons explored and demonstrated in this paper will find their applications in developing optical and electronic nanoscale switching devices.

\section{ACKNOWLEDGMENTS}

A.I. would like to acknowledge the funding provided by TRADA-51-82 PSC-CUNY Award No. 63061-00-51. G.G. appreciates financial support from the Air Force Research Laboratory (AFRL) through Grant No. FA9453-18-1-0100 and Award No. FA2386-18-1-0120. D.H. thanks the support from the Laboratory University Collaboration Initiative (LUCI) program and from the Air Force Office of Scientific Research (AFOSR). 


\section{APPENDIX A: PSEUDOSPIN-1 $\alpha$-DEPENDENT PAULI MATRICES}

In this paper, we mostly focus on dice lattice with $\phi=\pi / 4$ so the matrices in Eqs. (2) and (3) reduce to regular $3 \times 3$ Pauli matrices:

$$
\begin{gathered}
\hat{\Sigma}_{x}^{(1)}=\frac{1}{\sqrt{2}}\left[\begin{array}{ccc}
0 & 1 & 0 \\
1 & 0 & 1 \\
0 & 1 & 0
\end{array}\right], \\
\hat{\Sigma}_{y}^{(1)}=\frac{i}{\sqrt{2}}\left[\begin{array}{ccc}
0 & -1 & 0 \\
1 & 0 & -1 \\
0 & 1 & 0
\end{array}\right] .
\end{gathered}
$$

Sometimes, the third Pauli matrix,

$$
\hat{\Sigma}_{z}^{(1)}=\left[\begin{array}{ccc}
1 & 0 & 0 \\
0 & 0 & 0 \\
0 & 0 & -1
\end{array}\right]
$$

is also employed to introduce an energy gap for a pseudospin1 Hamiltonian [76].

Since all matrices, including additional interacting Hamiltonian terms derived in the next Appendix B, are Hermitian, it is convenient to introduce two new matrices

$$
\hat{\Sigma}_{ \pm}^{(1)}=\hat{\Sigma}_{x}^{(1)} \pm i \hat{\Sigma}_{y}^{(1)},
$$

which have the following structure:

$$
\begin{aligned}
& \hat{\Sigma}_{+}^{(1)}=\sqrt{2}\left[\begin{array}{ccc}
0 & \hat{\mathbb{I}}_{2} \\
0 & 0 \\
0 & 0 & 0
\end{array}\right]=\sqrt{2}\left[\begin{array}{lll}
0 & 1 & 0 \\
0 & 0 & 1 \\
0 & 0 & 0
\end{array}\right], \\
& \hat{\Sigma}_{-}^{(1)}=\sqrt{2}\left[\begin{array}{lll}
0 & 0 & 0 \\
\hat{\mathbb{I}}_{2} & 0 \\
& & 0
\end{array}\right]=\sqrt{2}\left[\begin{array}{lll}
0 & 0 & 0 \\
1 & 0 & 0 \\
0 & 1 & 0
\end{array}\right] .
\end{aligned}
$$

Here, $\hat{\mathbb{I}}_{2}$ is a $2 \times 2$ unit matrix corresponding to pseudospin$1 / 2$ system, i.e.,

$$
\hat{\mathbb{I}}_{2}=\left[\begin{array}{ll}
1 & 0 \\
0 & 1
\end{array}\right]
$$

Importantly, the obtained matrices $\hat{\Sigma}_{ \pm}^{(1)}$ satisfy the simple commutation relations $\left[\hat{\Sigma}_{+}^{(1)}, \hat{\Sigma}_{-}^{(1)}\right]=2 \hat{\Sigma}_{z}^{(1)}$ and $\left[\hat{\Sigma}_{z}^{(1)}, \hat{\Sigma}_{ \pm}^{(1)}\right]= \pm \hat{\Sigma}_{ \pm}^{(1)}$.

\section{APPENDIX B: HAMILTONIAN OF ELECTRONS IN DICE LATTICE WITH ARBITRARY DIRECTION FOR LIGHT POLARIZATION}

In contrast to the derived Eq. (12), we now consider an arbitrary direction for light polarization. We aim to obtain the dressed states of electrons in a dice lattice under linearly polarized light with its vector potential:

$$
\boldsymbol{A}^{(L)}\left(\xi_{0}, t\right)=\left[\begin{array}{l}
A_{x}^{(L)}\left(\xi_{0}, t\right) \\
A_{y}^{(L)}\left(\xi_{0}, t\right)
\end{array}\right]=\frac{\mathcal{E}_{0}}{\omega} \cos (\omega t)\left[\begin{array}{c}
\cos \xi_{0} \\
\sin \xi_{0}
\end{array}\right] .
$$

Particularly, the case for light polarization along the $x$ axis is simply obtained by setting $\xi_{0}=0$. In the presence of light irradiation, the new Hamiltonian is acquired by the standard substitution of $k_{x, y} \rightarrow k_{x, y}-(e / \hbar) A_{x, y}^{(L)}\left(\xi_{0}, t\right)$ in the noninteracting Hamiltonian for both components of the vector potential $\boldsymbol{A}^{(L)}\left(\xi_{0}, t\right)$.
Since the noninteracting Hamiltonian in the absence of irradiation is linear with respect to wave vector $\boldsymbol{k}$ of electrons, the effect of imposed irradiation can be included by

$$
\hat{\mathcal{H}}_{1, \tau}(\boldsymbol{k}) \Longrightarrow \hat{\mathbb{H}}^{(L)}(\boldsymbol{k}, t \mid \beta)=\hat{\mathcal{H}}_{1, \tau}(\boldsymbol{k})+\hat{\mathcal{H}}_{I}^{(L)}\left(\xi_{0}, t\right),
$$

where subscript-index 1 is associated with $\alpha=1$ for the dice lattice. Moreover, the interaction Hamiltonian term in Eq. (B2) is given by

$$
\mathbb{H}_{I}^{(L)}\left(\xi_{0}, t\right)=-\frac{\tau c_{0}}{\sqrt{2}} \cos (\omega t)\left[\begin{array}{ccc}
0 & e^{-i \tau \xi_{0}} & 0 \\
e^{i \tau \xi_{0}} & 0 & e^{-i \tau \xi_{0}} \\
0 & e^{i \tau \xi_{0}} & 0
\end{array}\right],
$$

where $\tau= \pm 1$ represents the valley index and the coupling constant $c_{0}=e v_{F} \mathcal{E}_{0} / \omega$ remains the same for all types of light polarizations. This implies that the light-polarization effect on energy dispersion of electrons becomes similar in magnitude but different in features.

Explicitly, the periodic time dependence in the interaction Hamiltonian term $\hat{\mathbb{H}}_{A}^{(L)}\left(\xi_{0}, t\right)$ in Eq. (B3) could be cast into the following form:

$$
\hat{\mathbb{H}}_{I}^{(L)}\left(\xi_{0}, t\right)=\hat{\mathbb{O}}_{1, \tau}\left(\xi_{0}\right) e^{i \omega t}+\hat{\mathbb{O}}_{1, \tau}^{\dagger}\left(\xi_{0}\right) e^{-i \omega t},
$$

where the operator $\hat{\mathbb{O}}_{1, \tau}\left(\xi_{0}\right)$ and its conjugate $\hat{\mathbb{O}}_{1, \tau}^{\dagger}\left(\xi_{0}\right)$ are time independent. Then, it is straightforward to find the operator $\hat{\mathbb{O}}_{1, \tau}\left(\xi_{0}\right)$ from Eq. (B3), yielding

$$
\hat{\mathbb{O}}_{1, \tau}\left(\xi_{0}\right)=-\frac{\tau c_{0}}{2 \sqrt{2}}\left[\begin{array}{ccc}
0 & e^{-i \tau \xi_{0}} & 0 \\
e^{i \tau \xi_{0}} & 0 & e^{-i \tau \xi_{0}} \\
0 & e^{i \tau \xi_{0}} & 0
\end{array}\right],
$$

and it is equivalent to Eq. (B3) except for the prefactor. Moreover, matrix $\hat{\mathbb{O}}_{1, \tau}\left(\xi_{0}\right)$ itself is Hermitian, which is unique for linearly polarized light but not for the case of any other types of elliptical polarization, including the circular one.

By using Eqs. (B2) and (B4), the effective timeindependent Hamiltonian under the high-frequency and off-resonant limits can be derived based on the standard Floquet-Magnus expansion approach, leading to

$$
\begin{aligned}
\hat{\mathcal{H}}_{\mathrm{eff}}= & \hat{\mathcal{H}}_{1, \tau}(\boldsymbol{k})+\frac{1}{\hbar \omega}\left[\hat{\mathbb{O}}_{1, \tau}, \hat{\mathbb{O}}_{1, \tau}^{\dagger}\right]+\frac{1}{2(\hbar \omega)^{2}} \\
& \times\left\{\left[\left[\hat{\mathbb{O}}_{1, \tau}, \hat{\mathcal{H}}_{1, \tau}(\boldsymbol{k})\right], \hat{\mathbb{O}}_{1, \tau}^{\dagger}\right]+\text { H.c. }\right\}+\cdots,
\end{aligned}
$$

where the first term in the expansion is just the noninteracting Hamiltonian, while the second term $\left[\hat{\mathbb{O}}_{1, \tau}, \hat{\mathbb{O}}_{1, \tau}^{\dagger}\right]$ is zero since matrix $\hat{\mathbb{O}}_{1, \tau}$ is Hermitian. However, this conclusion holds true only for linearly polarized light but not for any other types of polarization or a finite band gap. The third term $\hat{\mathbb{T}}_{2}\left(\lambda_{0} \mid k, \theta_{\mathbf{k}}\right)$ in Eq. (B6) for a dice lattice has been calculated as

$$
\begin{aligned}
& \hat{\mathbb{T}}_{2}\left(\lambda_{0} \mid k, \theta_{\mathbf{k}}\right) \\
& =i \frac{\lambda_{0}^{2}}{4 \sqrt{2} \hbar v_{F}\left\{k_{y} \cos \xi_{0}-\tau k_{x} \sin \left(\tau \xi_{0}\right)\right\}} \\
& \quad \times\left[\begin{array}{ccc}
0 & \exp \left(-i \tau \xi_{0}\right) & 0 \\
-\exp \left(i \tau \xi_{0}\right) & 0 & \exp \left(-i \tau \xi_{0}\right) \\
0 & -\exp \left(i \tau \xi_{0}\right) & 0
\end{array}\right]
\end{aligned}
$$




$$
\begin{aligned}
= & \frac{\lambda_{0}^{2}}{4 \sqrt{2}} \hbar v_{F}\left\{k_{y} \cos \xi_{0}-\tau k_{x} \sin \left(\tau \xi_{0}\right)\right\}\left\{\sin \left(\xi_{0} \tau\right) \hat{\Sigma}_{x}^{(1)}\right. \\
& \left.-\cos \xi_{0} \hat{\Sigma}_{y}^{(1)}\right\} .
\end{aligned}
$$

Here, we would like to emphasize that if the polarization direction of imposed radiation differs from the $x$ axis $\left(\xi_{0} \neq 0\right)$, there exists an additional $k_{x}$ related term in Eq. (B7) which leads to a discontinuity for electron tunneling at two boundaries of a barrier region. As a result, the boundary conditions for the components of dressed-state wave functions in a dice lattice must be modified accordingly.

\section{APPENDIX C: ANISOTROPIC ELECTRON DRESSED-STATE TUNNELING IN GRAPHENE AND A DICE LATTICE}

As one of the important consequences of finite anisotropy, we find that the directions of group velocity $\boldsymbol{V}_{G}$ and spinor vector $S$ are given by

$$
\begin{aligned}
\boldsymbol{S}^{\gamma}\left(\lambda_{0}, \boldsymbol{k}\right) & =\frac{\gamma}{\sqrt{k_{x}^{2}+\left[a_{0}\left(\lambda_{0}\right) k_{y}\right]^{2}}}\left[\begin{array}{c}
k_{x} \\
a_{0}\left(\lambda_{0}\right) k_{y}
\end{array}\right], \\
\boldsymbol{V}_{G}^{\gamma}\left(\lambda_{0}, \boldsymbol{k}\right) & =\frac{1}{\hbar}\left[\begin{array}{c}
\partial / \partial k_{x} \\
\partial / \partial k_{y}
\end{array}\right] \varepsilon_{0}^{\gamma}\left(\lambda_{0}, \boldsymbol{k}\right) \\
& =\frac{\gamma v_{F}}{\sqrt{k_{x}^{2}+\left[a_{0}\left(\lambda_{0}\right) k_{y}\right]^{2}}}\left[\begin{array}{c}
k_{x} \\
a_{0}^{2}\left(\lambda_{0}\right) k_{y}
\end{array}\right],
\end{aligned}
$$

which are aligned neither with each other nor with the electron wave vector $\boldsymbol{k}$. Here, vector $\boldsymbol{S}^{\gamma}$ is proportional to the spinor wave function in Eq. (23) and switches its direction to opposite one for hole states with $\gamma=-1$ in comparison with electron ones having $\gamma=+1$. Meanwhile, $\boldsymbol{V}_{G}^{\gamma}$ specifies the direction for incident particles. The angles of the two vectors in Eqs. (C1) and (C2) relative to the $x$ axis are determined by the relations $\tan \Theta_{\mathbf{S}}\left(\lambda_{0}\right)=\left(k_{y} / k_{x}\right) a_{0}\left(\lambda_{0}\right)$ and $\tan \Theta_{\mathbf{V}}\left(\lambda_{0}\right)=\left(k_{y} / k_{x}\right) a_{0}^{2}\left(\lambda_{0}\right)$ or, alternatively, $\tan \Theta_{\mathbf{V}}\left(\lambda_{0}\right)=$ $a_{0}\left(\lambda_{0}\right) \tan \Theta_{\mathbf{S}}\left(\lambda_{0}\right)=a_{0}^{2}\left(\lambda_{0}\right) \tan \theta_{\mathbf{k}}$.

The crystal long axes for anisotropic energy dispersion (or $\hat{\boldsymbol{x}}$ direction) and the normal direction of a potential barrier (or $\hat{\boldsymbol{x}}^{\prime}$ direction) are generally not aligned with each other. Therefore, we need to introduce two coordinate frames: $\{x, y\}$ for the $\hat{\boldsymbol{x}}$ vector while $\left\{x^{\prime}, y^{\prime}\right\}$ for the $\hat{\boldsymbol{x}}^{\prime}$ vector, as depicted in Fig. 3 in the main text.

In a similar way, the same incident-electron wave vector $\boldsymbol{k}$ can be decomposed either as $\left\{k_{x}, k_{y}\right\}$ or as $\left\{k_{x^{\prime}}, k_{y^{\prime}}\right\}$ in two different frames, but its magnitude $k$ should always be the same. These two frames are related to each other by an inplane rotation angle $\beta$, and the corresponding rotation matrix $\hat{\mathbb{R}}(\beta)$ is

$$
\hat{\mathbb{R}}(\beta)=\left[\begin{array}{cc}
\cos \beta & -\sin \beta \\
\sin \beta & \cos \beta
\end{array}\right] .
$$

Consequently, we arrive at $\theta_{\mathbf{k}}=\theta_{\mathbf{k}^{\prime}}+\beta$ or $\Theta_{\mathbf{V}}=\Theta_{V}^{\prime}+\beta$, and the wave vector $\boldsymbol{k}$ in these two reference frames are related by

$$
\left[\begin{array}{l}
k_{x} \\
k_{y}
\end{array}\right]=\hat{\mathbb{R}}(\beta)\left[\begin{array}{l}
k_{x^{\prime}} \\
k_{y^{\prime}}
\end{array}\right] .
$$

The obtained relation in Eq. (C4) remains true for both incident and reflected waves within the barrier and zero potential regions. The reason behind introducing another reference frame $\left\{x^{\prime}, y^{\prime}\right\}$ comes from the conservation of transverse wave number $k_{y^{\prime}}$ across the potential barrier for all regions.

We first note that all unknowns in Eqs. (27) are associated with both $\{x, y\}$ and $\left\{x^{\prime}, y^{\prime}\right\}$ reference frames. For the fixed kinetic energy $\mathcal{E}_{0}$ of an incident particle in region 1 , from Eq. (22) we find $\left\{k_{x}^{(1)}, k_{y}^{(1)}\right\}$ in the $\{x, y\}$ frame, i.e.,

$$
\left[k_{x}^{(1)}\right]^{2}+\left[a_{0}\left(\lambda_{0}\right) k_{y}^{(1)}\right]^{2}=\left(\frac{\mathcal{E}_{0}}{\hbar v_{F}}\right)^{2} .
$$

In region 2 with a finite potential barrier $V_{B}$, we obtain a similar relation between the wave-vector components $k_{x, y}^{(2)}$ and the anisotropy $a_{0}\left(\lambda_{0}\right)$, except that the incoming particle energy $\mathcal{E}_{0}$ is replaced by $\mathcal{E}_{0}-V_{B}$. Knowing $\left\{k_{x}^{(1)}, k_{y}^{(1)}\right\}$ and $\left\{k_{x}^{(2)}, k_{y}^{(2)}\right\}$ in the $\{x, y\}$ frame, we are able to determine $\Theta_{\mathbf{S}}^{(1)}$ and $\Theta_{\mathrm{S}}^{(2)}$ from the angle definitions of spinor and group velocity vectors, involving energy-dispersion anisotropy.

Physically, it is the group velocity component $V_{G, x^{\prime}}^{\gamma}$ or its angle $\Theta_{V}^{\prime}$ within the $\left\{x^{\prime}, y^{\prime}\right\}$ reference frame that determines the direction of a moving wave [71]. We know that there exist two solutions within the $\left\{x^{\prime}, y^{\prime}\right\}$ frame from Eq. (C5) in both region 1 and region 2, corresponding to the forward $\left(V_{G, x^{\prime}}^{\gamma}>0\right)$ and backward $\left(V_{G, x^{\prime}}^{\gamma}<0\right)$ moving waves, respectively. The frame-rotation matrix in Eq. (C3) can project these found solutions for $\left\{k_{x^{\prime}}^{(1,2)}, k_{y^{\prime}}^{(1,2)}\right\}_{ \pm}$back to $\left\{k_{x,}^{(1,2)}, k_{y}^{(1,2)}\right\}_{ \pm}$in the $\{x, y\}$ frame, from which the spinor angles $\Theta_{\mathbf{S}, \pm}^{(1)}$ and $\Theta_{\mathbf{S}, \pm}^{(1, r)}$ in region 1, as well as $\Theta_{\mathbf{S}, \pm}^{(2)}$ and $\Theta_{\mathbf{S}, \pm}^{(2, r)}$ in region 2, can be computed.

Two unknown components of the same group velocity vector $\boldsymbol{V}_{G}^{\gamma}\left(\lambda_{0}, \boldsymbol{k}\right)$ in the $\left\{x^{\prime}, y^{\prime}\right\}$ frame can be obtained from its two known components in the $\{x, y\}$ frame by using Eq. (C4), yielding

$$
\begin{aligned}
{\left[\begin{array}{c}
V_{G, x^{\prime}}^{\gamma}\left(\lambda_{0}, \boldsymbol{k}\right) \\
V_{G, y^{\prime}}^{\gamma}\left(\lambda_{0}, \boldsymbol{k}\right)
\end{array}\right] } & =\hat{\mathbb{R}}(-\beta)\left[\begin{array}{c}
V_{G, x}^{\gamma}\left(\lambda_{0}, \boldsymbol{k}\right) \\
V_{G, y}^{\gamma}\left(\lambda_{0}, \boldsymbol{k}\right)
\end{array}\right] \\
& =\frac{\gamma v_{F}}{\sqrt{k_{x}^{2}+\left[a_{0}\left(\lambda_{0}\right) k_{y}\right]^{2}}} \hat{\mathbb{R}}(-\beta)\left[\begin{array}{c}
k_{x} \\
a_{0}^{2}\left(\lambda_{0}\right) k_{y}
\end{array}\right] .
\end{aligned}
$$

We solve Eq. (C6) in conjunction with Eq. (C5) for $V_{G, x^{\prime}}^{\gamma}\left(\lambda_{0}, \boldsymbol{k}\right)$ and express two solutions explicitly in terms of the known $k_{y^{\prime}}$ as

$$
\begin{aligned}
V_{G, x^{\prime}}^{\gamma}\left(\lambda_{0}, \boldsymbol{k} \mid \beta\right)= & \pm \gamma \frac{v_{F}}{\sqrt{2}}\left\{1+a_{0}^{2}\left(\lambda_{0}\right)+\left[1-a_{0}^{2}\left(\lambda_{0}\right)\right] \cos (2 \beta)\right. \\
& \left.-2\left[\frac{\hbar v_{F}}{\mathcal{E}_{0}} a_{0}\left(\lambda_{0}\right) k_{y^{\prime}}\right]^{2}\right\}^{1 / 2}, \quad \text { (C7) }
\end{aligned}
$$

which have opposite signs but equal magnitudes, indicating forward (+) and backward (-) waves, respectively. We emphasize that the relation in Eq. (C7) does not apply to wave-vector components, such as $k_{x}$ or $k_{x^{\prime}}$, as demonstrated in Fig. 4. In the absence of rotation $(\beta=0)$ between two frames, 
Eq. (C7) reduces to

$$
V_{G, x^{\prime}}^{\gamma}\left(\lambda_{0}, \boldsymbol{k} \mid \beta \rightarrow 0\right)= \pm \gamma v_{F} \sqrt{1-\left[\frac{\hbar v_{F}}{\mathcal{E}_{0}} a_{0}\left(\lambda_{0}\right) k_{y^{\prime}}\right]^{2}}
$$

or

$$
V_{G, x^{\prime}}^{\gamma}\left(\lambda_{0} \rightarrow 0, k \mid \beta\right)= \pm \gamma v_{F} \sqrt{1-\left(\frac{\hbar v_{F}}{\mathcal{E}_{0}} k_{y^{\prime}}\right)^{2}}
$$

if the electron-light interaction and anisotropy are turned off with $\lambda_{0}=0$, which is independent of angle $\beta$.

\section{APPENDIX D: BOUNDARY CONDITIONS FOR ANISOTROPIC HAMILTONIAN}

For a pseudospin-1 dice lattice including a barrier region, we address relevant boundary conditions for the case with an anisotropic Dirac cone and noncollinear $k_{x}$ and $k_{x^{\prime}}$ axes. In contrast to graphene, we find that the boundary conditions for a dice lattice change significantly with a finite anisotropy in energy dispersion.

We begin with the anisotropic pseudospin-1/2 graphene Hamiltonian, given by

$$
\hat{\mathcal{H}}_{0}\left(\lambda_{0}, \boldsymbol{k}\right)=\hbar v_{F}\left(\hat{\Sigma}_{x}^{(1 / 2)} k_{x}+a_{0}\left(\lambda_{0}\right) \hat{\Sigma}_{y}^{(1 / 2)} k_{y}\right),
$$

where $\hat{\Sigma}_{x}^{(1 / 2)}$ and $\hat{\Sigma}_{y}^{(1 / 2)}$ are the $2 \times 2$ Pauli matrices, related to Eqs. (A1) and (A2). Here, we consider two frames, $\{x, y\}$ and $\left\{x^{\prime}, y^{\prime}\right\}$, in which the former relates to the long axis of an elliptical energy dispersion for dressed states of electrons while the latter to the normal direction of a potential barrier. As a result, the decomposition of a wave vector $\boldsymbol{k}$ in these two frames can be written as $\left\{k_{x}, k_{y}\right\}$ or $\left\{k_{x^{\prime}}, k_{y^{\prime}}\right\}$, respectively, which are related to each other by a rotation matrix $\hat{\mathbb{R}}(\beta)$ in Eq. (C4). To find the proper boundary conditions, we need to transform the $k_{x, y}$-dependent Hamiltonian into the $\left\{x^{\prime}, y^{\prime}\right\}$ frame, integrate each of relevant equations over a small interval from $-\delta x^{\prime}$ to $\delta x^{\prime}$, and take the limit of $\delta x^{\prime} \rightarrow 0$ afterwards $[42,43]$.

Let us start with the transformed dressed-state Hamiltonian for anisotropic graphene within the $\left\{x^{\prime}, y^{\prime}\right\}$ frame, given by

$$
\hat{\mathcal{H}}_{0}\left(\lambda_{0}, \boldsymbol{k}\right)=\hbar v_{F} k\left[\begin{array}{cc}
0 & \cos \left(\theta_{\mathbf{k}}^{\prime}+\beta\right)-i a_{0}\left(\lambda_{0}\right) \sin \left(\theta_{\mathbf{k}}^{\prime}+\beta\right) \\
\cos \left(\theta_{\mathbf{k}}^{\prime}+\beta\right)+i a_{0}\left(\lambda_{0}\right) \sin \left(\theta_{\mathbf{k}}^{\prime}+\beta\right) & 0
\end{array}\right.
$$

where $\theta_{\mathbf{k}}=\theta_{\mathbf{k}}^{\prime}+\beta, \tan \theta_{\mathbf{k}}=k_{y} / k_{x}$. For the case with $a_{0}\left(\lambda_{0}\right)=1$, the transformed Hamiltonian in Eq. (D2) within the $\left\{x^{\prime}, y^{\prime}\right\}$ is simplified to

$$
\hat{\mathcal{H}}_{0}\left(\lambda_{0}, \boldsymbol{k}\right)=\hbar v_{F}\left[\begin{array}{cc}
0 & k_{-} e^{-i \beta} \\
k_{+} e^{i \beta} & 0
\end{array}\right],
$$

where $k_{ \pm}=k_{x^{\prime}} \pm i k_{y^{\prime}}$. Since the discontinuity related to $\partial / \partial x^{\prime}$, due to the existence of potential barrier, is associated with the $x^{\prime}$ coordinate, by using $k_{x^{\prime}} \rightarrow-i \partial / \partial x^{\prime}$ we generalize the Hamiltonian in Eq. (D3) into

$$
\hat{\mathcal{H}}_{0}\left(\lambda_{0} \mid x^{\prime}, k_{y}^{\prime}\right)=\hbar v_{F}\left[\begin{array}{cc}
0 & \left(-i \partial / \partial x^{\prime}-i k_{y^{\prime}}\right) e^{-i \beta} \\
\left(-i \partial / \partial x^{\prime}+i k_{y^{\prime}}\right) e^{i \beta} & 0
\end{array}\right],
$$

while all other continuous terms on both sides of the eigenvalue equation approach zero in the limit of $\delta x^{\prime} \rightarrow 0$, i.e.,

$$
\int_{-\delta x}^{\delta x} V_{B} \Theta(x) \varphi_{j}(x) \rightarrow 0, \quad \int_{-\delta x}^{\delta x} \mathcal{E}_{0} \varphi_{j}(x) \rightarrow 0,
$$

where $\varphi_{j}(x)$ with $j=1,2$ represents one of the two wave-function components. As a result, only the terms containing $k_{x^{\prime}} \rightarrow$ $-i \partial / \partial x^{\prime}$ make nonzero contributions to the boundary conditions, leading to

$$
\begin{aligned}
& \int_{-\delta x}^{\delta x}-i \frac{\partial}{\partial x^{\prime}}\left[\cos \beta-i a_{0}\left(\lambda_{0}\right) \sin \beta\right] \varphi_{2}\left(x^{\prime}\right)=0 \rightarrow \varphi_{2}\left(\delta x^{\prime}\right)=\varphi_{2}\left(-\delta x^{\prime}\right), \\
& \int_{-\delta x}^{\delta x}-i \frac{\partial}{\partial x^{\prime}}\left[\cos \beta+i a_{0}\left(\lambda_{0}\right) \sin \beta\right] \varphi_{1}\left(x^{\prime}\right)=0 \rightarrow \varphi_{1}\left(\delta x^{\prime}\right)=\varphi_{1}\left(-\delta x^{\prime}\right) .
\end{aligned}
$$

These obtained results are equivalent to those for the earlier considered isotropic graphene, therefore, the anisotropy and the rotation $\hat{\mathbb{R}}(\beta)$ do not affect boundary conditions.

The situation changes drastically for a dice lattice with the pseudospin-1 Hamiltonian. We once again rewrite the Hamiltonian in Eq. (1) within the $\left\{x^{\prime}, y^{\prime}\right\}$ frame, leaving out all the continuous terms involving kinetic energy $\mathcal{E}_{0}$, piecewise potential $V_{B} \Theta(x)$, and constant $k_{y^{\prime}}$. As a result, we only keep the terms including $-i \partial / \partial x^{\prime}$ and are left with

$$
\hat{\mathcal{H}}_{1}^{\tau}\left(x^{\prime}\right)=\frac{\hbar v_{F}}{\sqrt{2}}\left(-i \frac{\partial}{\partial x^{\prime}}\right)\left\{\left[\begin{array}{ccc}
0 & \tau \cos \beta-i a_{1}\left(\lambda_{0}\right) \sin \beta & 0 \\
0 & 0 & \tau \cos \beta-i a_{1}\left(\lambda_{0}\right) \sin \beta \\
0 & 0 & 0
\end{array}\right]+\text { H.c. }\right\} .
$$


Correspondingly, the boundary conditions are found to be

$$
\varphi_{2}\left(-\delta x^{\prime}\right)=\varphi_{2}\left(\delta x^{\prime}\right), \quad c_{\tau}^{+}\left(\lambda_{0}, \beta\right) \varphi_{1}\left(-\delta x^{\prime}\right)+c_{\tau}^{-}\left(\lambda_{0}, \beta\right) \varphi_{3}\left(-\delta x^{\prime}\right)=c_{\tau}^{+}\left(\lambda_{0}, \beta\right) \varphi_{1}\left(\delta x^{\prime}\right)+c_{\tau}^{-}\left(\lambda_{0}, \beta\right) \varphi_{3}\left(\delta x^{\prime}\right),
$$

where

$$
c_{\tau}^{ \pm}\left(\lambda_{0}, \beta\right)=\tau \cos \beta \pm i a_{1}\left(\lambda_{0}\right) \sin \beta .
$$

In the case with $a_{1}\left(\lambda_{0}\right)=0$ and collinear $x$ and $x^{\prime}(\beta=0), c_{\tau}^{ \pm}\left(\lambda_{0}, \beta=0\right)=\tau$, and then we immediately recover the previously obtained boundary conditions for a dice lattice [43]:

$$
\varphi_{2}(-\delta x)=\varphi_{2}(\delta x), \quad \varphi_{1}(-\delta x)+\varphi_{3}(-\delta x)=\varphi_{1}(\delta x)+\varphi_{3}(\delta x) .
$$

For an isotropic Dirac cone but with $\beta \neq 0\left(k_{x} \neq k_{x^{\prime}}\right), c_{\tau}^{ \pm}\left(\lambda_{0} \rightarrow 0, \beta\right) \rightarrow \tau e^{ \pm i \tau \beta}$, and the boundary conditions must be modified accordingly.

[1] E. Illes, Properties of the $\alpha-T_{3}$ model, Ph.D. thesis, University of Guelph, Canada, 2017.

[2] K. Novoselov, A. K. Geim, S. Morozov, D. Jiang, M. Katsnelson, I. Grigorieva, S. Dubonos, and A. Firsov, Twodimensional gas of massless Dirac fermions in graphene, Nature 438, 197 (2005).

[3] A. H. Castro Neto, F. Guinea, N. M. R. Peres, K. S. Novoselov, and A. K. Geim, The electronic properties of graphene, Rev. Mod. Phys. 81, 109 (2009).

[4] A. D. Kovács, G. Dávid, B. Dóra, and J. Cserti, Frequencydependent magneto-optical conductivity in the generalized $\alpha-$ $t_{3}$ model, Phys. Rev. B 95, 035414 (2017).

[5] J. D. Malcolm and E. H. Nicol, Frequency-dependent polarizability, plasmons, and screening in the two-dimensional pseudospin-1 dice lattice, Phys. Rev. B 93, 165433 (2016).

[6] J. Vidal, R. Mosseri, and B. Douçot, Aharonov-Bohm Cages in Two-Dimensional Structures, Phys. Rev. Lett. 81, 5888 (1998).

[7] J. Vidal, P. Butaud, B. Douçot, and R. Mosseri, Disorder and interactions in Aharonov-Bohm cages, Phys. Rev. B 64, 155306 (2001).

[8] O. Kibis, Metal-insulator transition in graphene induced by circularly polarized photons, Phys. Rev. B 81, 165433 (2010).

[9] A. Iurov, G. Gumbs, and D. Huang, Peculiar electronic states, symmetries, and Berry phases in irradiated $\alpha-t_{3}$ materials, Phys. Rev. B 99, 205135 (2019).

[10] R. A. Vicencio, C. Cantillano, L. Morales-Inostroza, B. Real, C. Mejía-Cortés, S. Weimann, A. Szameit, and M. I. Molina, Observation of Localized States in Lieb Photonic Lattices, Phys. Rev. Lett. 114, 245503 (2015).

[11] S. Mukherjee, A. Spracklen, D. Choudhury, N. Goldman, P. Öhberg, E. Andersson, and R. R. Thomson, Observation of a Localized Flat-Band State in a Photonic Lieb Lattice, Phys. Rev. Lett. 114, 245504 (2015).

[12] J. Romhányi, K. Penc, and R. Ganesh, Hall effect of triplons in a dimerized quantum magnet, Nat. Commun. 6, 6805 (2015).

[13] X. Huang, Y. Lai, Z. H. Hang, H. Zheng, and C. Chan, Dirac cones induced by accidental degeneracy in photonic crystals and zero-refractive-index materials, Nat. Mater. 10, 582 (2011).

[14] Y. Li, S. Kita, P. Muñoz, O. Reshef, D. I. Vulis, M. Yin, M. Lončar, and E. Mazur, On-chip zero-index metamaterials, Nat. Photonics 9, 738 (2015).

[15] W.-X. Qiu, S. Li, J.-H. Gao, Y. Zhou, and F.-C. Zhang, Designing an artificial Lieb lattice on a metal surface, Phys. Rev. B 94, 241409 (2016).
[16] L. Santos, M. A. Baranov, J. I. Cirac, H.-U. Everts, H. Fehrmann, and M. Lewenstein, Atomic Quantum Gases in Kagomé Lattices, Phys. Rev. Lett. 93, 030601 (2004).

[17] J. Ruostekoski, Optical Kagome Lattice for Ultracold Atoms with Nearest Neighbor Interactions, Phys. Rev. Lett. 103, 080406 (2009).

[18] G.-B. Jo, J. Guzman, C. K. Thomas, P. Hosur, A. Vishwanath, and D. M. Stamper-Kurn, Ultracold Atoms in a Tunable Optical Kagome Lattice, Phys. Rev. Lett. 108, 045305 (2012).

[19] T. Baba, Slow light in photonic crystals, Nat. Photonics 2, 465 (2008).

[20] D. Leykam, A. Andreanov, and S. Flach, Artificial flat band systems: From lattice models to experiments, Adv. Phys.: X 3, 1473052 (2018).

[21] B. Dey and T. K. Ghosh, Photoinduced valley and electron-hole symmetry breaking in $\alpha-t_{3}$ lattice: The role of a variable Berry phase, Phys. Rev. B 98, 075422 (2018).

[22] B. Dey and T. K. Ghosh, Floquet topological phase transition in the $\alpha-t_{3}$ lattice, Phys. Rev. B 99, 205429 (2019).

[23] B. Dey, P. Kapri, O. Pal, and T. K. Ghosh, Unconventional phases in a Haldane model of dice lattice, Phys. Rev. B 101, 235406 (2020).

[24] D. Bercioux, N. Goldman, and D. F. Urban, Topology-induced phase transitions in quantum spin Hall lattices, Phys. Rev. A 83, 023609 (2011)

[25] B. Dóra, J. Kailasvuori, and R. Moessner, Lattice generalization of the Dirac equation to general spin and the role of the flat band, Phys. Rev. B 84, 195422 (2011).

[26] M. Rizzi, V. Cataudella, and R. Fazio, Phase diagram of the bose-hubbard model with $\mathscr{T}_{3}$ symmetry, Phys. Rev. B 73 , 144511 (2006).

[27] M. Vigh, L. Oroszlány, S. Vajna, P. San-Jose, G. Dávid, J. Cserti, and B. Dóra, Diverging dc conductivity due to a flat band in a disordered system of pseudospin-1 Dirac-Weyl fermions, Phys. Rev. B 88, 161413(R) (2013).

[28] SK Firoz Islam and P. Dutta, Valley-polarized magnetoconductivity and particle-hole symmetry breaking in a periodically modulated $\alpha-t_{3}$ lattice, Phys. Rev. B 96, 045418 (2017).

[29] SK Firoz Islam and A. Saha, Driven conductance of an irradiated semi-Dirac material, Phys. Rev. B 98, 235424 (2018).

[30] F. Wang and Y. Ran, Nearly flat band with Chern number $\mathrm{c}=2$ on the dice lattice, Phys. Rev. B 84, 241103(R) (2011).

[31] A. Iurov, G. Gumbs, and D. Huang, Many-body effects and optical properties of single and double layer $\alpha$-lattices, J. Phys. Condens. Matter 32, 415303 (2020). 
[32] T. Biswas and T. K. Ghosh, Dynamics of a quasiparticle in the $\alpha-t_{3}$ model: Role of pseudospin polarization and transverse magnetic field on zitterbewegung, J. Phys.: Condens. Matter 30, 075301 (2018).

[33] T. Biswas and T. K. Ghosh, Magnetotransport properties of the $\alpha-T_{3}$ model, J. Phys.: Condens. Matter 28, 495302 (2016).

[34] E. Illes and E. J. Nicol, Magnetic properties of the $\alpha-T_{3}$ model: Magneto-optical conductivity and the Hofstadter butterfly, Phys. Rev. B 94, 125435 (2016).

[35] E. Illes, J. P. Carbotte, and E. J. Nicol, Hall quantization and optical conductivity evolution with variable Berry phase in the $\alpha-t_{3}$ model, Phys. Rev. B 92, 245410 (2015).

[36] A. Raoux, M. Morigi, J.-N. Fuchs, F. Piéchon, and G. Montambaux, From Dia- to Paramagnetic Orbital Susceptibility of Massless Fermions, Phys. Rev. Lett. 112, 026402 (2014).

[37] F. Piéchon, J. Fuchs, A. Raoux, and G. Montambaux, Tunable orbital susceptibility in $\alpha-t_{3}$ tight-binding models, J. Phys.: Conf. Ser. 603, 012001 (2015).

[38] D. Huang, A. Iurov, H.-Y. Xu, Y.-C. Lai, and G. Gumbs, Interplay of Lorentz-Berry forces in position-momentum spaces for valley-dependent impurity scattering in $\alpha-t_{3}$ lattices, Phys. Rev. B 99, 245412 (2019).

[39] J. Wang, J. F. Liu, and C. S. Ting, Recovered minimal conductivity in the $\alpha-t_{3}$ model, Phys. Rev. B 101, 205420 (2020).

[40] T. Louvet, P. Delplace, A. A. Fedorenko, and D. Carpentier, On the origin of minimal conductivity at a band crossing, Phys. Rev. B 92, 155116 (2015).

[41] A. Iurov, L. Zhemchuzhna, D. Dahal, G. Gumbs, and D. Huang, Quantum-statistical theory for laser-tuned transport and optical conductivities of dressed electrons in $\alpha-\mathscr{T}_{3}$ materials, Phys. Rev. B 101, 035129 (2020).

[42] E. Illes and E. J. Nicol, Klein tunneling in the $\alpha-t_{3}$ model, Phys. Rev. B 95, 235432 (2017).

[43] D. F. Urban, D. Bercioux, M. Wimmer, and W. Häusler, Barrier transmission of Dirac-like pseudospin-one particles, Phys. Rev. B 84, 115136 (2011)

[44] F. Anwar, A. Iurov, D. Huang, G. Gumbs, and A. Sharma, Interplay between effects of barrier tilting and scatterers within a barrier on tunneling transport of Dirac electrons in graphene, Phys. Rev. B 101, 115424 (2020).

[45] D. Dahal and G. Gumbs, Effect of energy band gap in graphene on negative refraction through the Veselago lens and electron conductance, J. Phys. Chem. Solids 100, 83 (2017).

[46] M. Katsnelson, K. Novoselov, and A. Geim, Chiral tunneling and the Klein paradox in graphene, Nat. Phys. 2, 620 (2006).

[47] M. Katsnelson and K. Novoselov, Graphene: New bridge between condensed matter physics and quantum electrodynamics, Solid State Commun. 143, 3 (2007).

[48] M. Barbier, P. Vasilopoulos, and F. M. Peeters, Extra Dirac points in the energy spectrum for superlattices on single-layer graphene, Phys. Rev. B 81, 075438 (2010).

[49] M. Barbier, P. Vasilopoulos, and F. M. Peeters, Single-layer and bilayer graphene superlattices: Collimation, additional Dirac points and Dirac lines, Philos. Trans. R. Soc. A 368, 5499 (2010).

[50] M. R. Masir, P. Vasilopoulos, and F. Peeters, Kronig-Penney model of scalar and vector potentials in graphene, J. Phys.: Condens. Matter 22, 465302 (2010).
[51] Z. Lan, N. Goldman, A. Bermudez, W. Lu, and P. Öhberg, Dirac-Weyl fermions with arbitrary spin in two-dimensional optical superlattices, Phys. Rev. B 84, 165115 (2011).

[52] Z. Lan, N. Goldman, and P. Öhberg, Coexistence of spin-1/2 and spin-1 Dirac-Weyl fermions in the edge-centered honeycomb lattice, Phys. Rev. B 85, 155451 (2012).

[53] P. M. Perez-Piskunow, G. Usaj, C. A. Balseiro, and L. E. F. Torres, Floquet chiral edge states in graphene, Phys. Rev. B 89 , 121401(R) (2014).

[54] H. L. Calvo, H. M. Pastawski, S. Roche, and L. E. F. Torres, Tuning laser-induced band gaps in graphene, Appl. Phys. Lett. 98, 232103 (2011).

[55] E. Suarez Morell and L. E. F. Foa Torres, Radiation effects on the electronic properties of bilayer graphene, Phys. Rev. B 86 , 125449 (2012).

[56] S. Morina, O. V. Kibis, A. A. Pervishko, and I. A. Shelykh, Transport properties of a two-dimensional electron gas dressed by light, Phys. Rev. B 91, 155312 (2015).

[57] N. Goldman and J. Dalibard, Periodically Driven Quantum Systems: Effective Hamiltonians and Engineered Gauge Fields, Phys. Rev. X 4, 031027 (2014).

[58] V. Dal Lago, E. Suarez Morell, and L. E. F. Foa Torres, Oneway transport in laser-illuminated bilayer graphene: A Floquet isolator, Phys. Rev. B 96, 235409 (2017).

[59] S. Morina, K. Dini, I. V. Iorsh, and I. A. Shelykh, Optical trapping of electrons in graphene, ACS Photonics 5, 1171 (2018).

[60] K. Kristinsson, O. Kibis, S. Morina, and I. Shelykh, Control of electronic transport in graphene by electromagnetic dressing, Sci. Rep. 6, 20082 (2016).

[61] O. V. Kibis, K. Dini, I. V. Iorsh, and I. A. Shelykh, All-optical band engineering of gapped Dirac materials, Phys. Rev. B 95, 125401 (2017).

[62] V. Shahnazaryan, V. Kozin, I. Shelykh, I. Iorsh, and O. Kyriienko, Tunable optical nonlinearity for TMD polaritons dressed by a Fermi sea, Phys. Rev. B 102, 115310 (2020).

[63] A. Iurov, L. Zhemchuzhna, G. Gumbs, and D. Huang, Exploring interacting Floquet states in black phosphorus: Anisotropy and bandgap laser tuning, J. Appl. Phys. 122, 124301 (2017).

[64] O. Kyriienko and A. S. Sørensen, Floquet Quantum Simulation with Superconducting Qubits, Phys. Rev. Appl. 9, 064029 (2018).

[65] O. Kibis, O. Kyriienko, and I. Shelykh, Structure of surface electronic states in strained mercury telluride, New J. Phys. 21, 043016 (2019).

[66] S. K. Firoz Islam and A. A. Zyuzin, Photoinduced interfacial chiral modes in threefold topological semimetal, Phys. Rev. B 100, 165302 (2019).

[67] A. Iurov, G. Gumbs, O. Roslyak, and D. Huang, Anomalous photon-assisted tunneling in graphene, J. Phys.: Condens. Matter 24, 015303 (2011).

[68] X. Ye, S.-S. Ke, X.-W. Du, Y. Guo, and H.-F. Lü, Quantum tunneling in the $\alpha-t_{3}$ model with an effective mass term, J. Low Temp. Phys. 199, 1332 (2020).

[69] A. Iurov, G. Gumbs, D. Huang, and L. Zhemchuzhna, Controlling plasmon modes and damping in buckled two-dimensional material open systems, J. Appl. Phys. 121, 084306 (2017).

[70] A. Iurov, G. Gumbs, and D. Huang, Temperature-and frequency-dependent optical and transport conductivities in doped buckled honeycomb lattices, Phys. Rev. B 98, 075414 (2018). 
[71] Z. Li, T. Cao, M. Wu, and S. G. Louie, Generation of anisotropic massless Dirac fermions and asymmetric Klein tunneling in few-layer black phosphorus superlattices, Nano Lett. 17, 2280 (2017).

[72] J. C. Sandoval-Santana, V. G. Ibarra-Sierra, A. Kunold, and G. G. Naumis, Floquet spectrum for anisotropic and tilted Dirac materials under linearly polarized light at all field intensities, J. Appl. Phys. 127, 234301 (2020).

[73] N. Stander, B. Huard, and D. Goldhaber-Gordon, Evidence for Klein Tunneling in Graphene p-n Junctions, Phys. Rev. Lett. 102, 026807 (2009).
[74] J. Dauber, K. J. Reijnders, L. Banszerus, A. Epping, K. Watanabe, T. Taniguchi, M. I. Katsnelson, F. Hassler, and C. Stampfer, Exploiting Aharonov-Bohm oscillations to probe Klein tunneling in tunable pn-junctions in graphene, arXiv:2008.02556.

[75] See Supplemental Material at http://link.aps.org/supplemental/ 10.1103/PhysRevResearch.2.043245 for some other secondary but yet very important cases for electron tunneling .

[76] E. V. Gorbar, V. P. Gusynin, and D. O. Oriekhov, Electron states for gapped pseudospin-1 fermions in the field of a charged impurity, Phys. Rev. B 99, 155124 (2019). 